\title{
Multimodal integration of diffusion MRI for better characterization of tissue biology
}

Chunlei Liu and Evren Özarslan

The self-archived postprint version of this journal article is available at Linköping University Institutional Repository (DiVA):

http:/ / urn.kb.se/ resolve?urn=urn:nbn:se:liu:diva- 155993

N.B.: When citing this work, cite the original publication.

Liu, C., Özarslan, E., (2019), Multimodal integration of diffusion MRI for better characterization of tissue biology, NMR in Biomedicine, 32(4). https:// doi.org/ 10.1002/nbm.3939

Original publication available at:

https:/ / doi.org/ 10.1002/ nbm.3939

Copyright: Wiley (12 months)

http:/ / eu.wiley.com/WileyCDA/ 


\title{
Multimodal integration of diffusion MRI for better characterization of tissue biology
}

\author{
Chunlei Liu' ${ }^{1,2}$ Evren Özarslan ${ }^{3,4}$
}

1. Department of Electrical Engineering and Computer Sciences, University of California, Berkeley, CA 94720, USA

2. Helen Wills Neuroscience Institute, University of California, Berkeley, CA 94720, USA

3. Department of Biomedical Engineering, Linköping University, Linköping, Sweden

4. Center for Medical Image Science and Visualization (CMIV), Linköping University, Linköping, Sweden

Running Title: Multimodal integration of diffusion MRI

\section{Corresponding addresses:}

Chunlei Liu, Ph.D.

505 Cory Hall, MC\# 1770

University of California, Berkeley, CA 94720, USA

Email: chunlei.liu@berkeley.edu

Evren Özarslan, Ph.D.

Linköping University

Linköping, Sweden

Email: evren.ozarslan@liu.se 


\section{Abbreviations}

MRI Magnetic resonance imaging

NMR Nuclear magnetic resonance

DTI Diffusion tensor imaging

PDF Probability density function

MAP Mean apparent propagator

RTOP Return-to-the origin probability

STI Susceptibility tensor imaging

QSM Quantitative susceptibility mapping

STIFT Structure tensor informed fiber tractography

DDIF Diffusion in the internal field

DR-COSY Diffusion-relaxation correlation spectroscopy

CPMG Carr-Purcell-Meiboom-Gill

ADC Apparent diffusion coefficient

BOLD Blood-oxygen-level-dependent

fMRI Functional magnetic resonance imaging 


\section{Abstract}

The contrast in diffusion-weighted MR images is due to variations of diffusion properties within the examined specimen. Certain microstructural information of the underlying tissues can be inferred through quantitative analyses of the diffusion-sensitized MR signals. In the first part of the paper, we review two types of approaches for characterizing diffusion MRI signals: Bloch's equations with diffusion terms and statistical descriptions. Specifically, we discuss expansions in terms of cumulants and orthogonal basis functions, the confinement tensor formalism, and tensor distribution models. Further insights of the tissue property may be obtained by integrating diffusion MRI with other techniques, which is the subject of the second part of the paper. We review examples involving magnetic susceptibility, structural tensors, internal field gradients, transverse relaxation and functional MRI. Integrating information provided by other imaging modalities (MR-based or otherwise) could be a key to improve our understanding of how diffusion MRI relates to physiology and biology. 


\section{Introduction}

The diffusion of water molecules (or other molecules in general) can be measured by MRI, typically through pulsed field gradients. This measurement is possible because the random movement of water molecules in an inhomogeneous magnetic field results in a random accumulation of phase angles thus an irreversible loss of phase coherence within an ensemble. In the literature, two approaches are commonly used to analyze the effect of diffusion on MR signals: one by solving the Bloch-Torrey equations and the other by statistical analysis. The BlochTorrey equations extend the Bloch equations by adding a diffusion term which phenomenologically characterizes a signal decay rate given by Fick's second law. On the other hand, statistical approaches treat the molecular movement as a random process which accrues a random phase due to the random motion in an inhomogeneous field. The effect of diffusion is characterized by the transitional probability density function.

However, the complexity of biological tissues dictates that knowing the diffusion coefficients or the statistics of the diffusion process may not be fully satisfactory for understanding the underlying biological structures and processes. A better understanding of the diffusion properties measured by MRI can be supplemented by models of the underlying tissue microstructures and by other complementary tissue properties, for example, magnetic susceptibility, relaxivity, incoherent flow and magnetization transfer. Some of these properties may affect diffusion measurements directly while all reflect aspects of the underlying tissues that are measured by diffusion MRI. The purpose of this review is to summarize multimodal approaches for characterizing and utilizing diffusion MRI for quantifying tissue properties. The reviewed approaches include various models for describing diffusion MRI signal and 
complementary MRI techniques that can be integrated with diffusion MRI techniques for improving the characterization of tissue properties. The scope is limited with the intention to complement other reviews within this special issue.

\section{Approaches for analyzing diffusion MRI signal}

The effect of translational molecular diffusion on MRI signal has been analyzed with various approaches including solving Bloch's equation with diffusion terms, stochastic ensemble averaging and microstructural modelling.

\subsection{Bloch's equation with diffusion terms}

In the case of spin echoes, the MR signals are not only attenuated by $\mathrm{T} 1$ and $\mathrm{T} 2$ relaxation, but also attenuated due to the diffusion of the molecules in inhomogeneous local magnetic field induced by internal magnetic susceptibilities or external field gradients. In the classical paper "spin echoes", Hahn stated that "the effect of self-diffusion will be qualitatively accounted for by using Bloch's equations with a diffusion term added" (1). Torrey provided a general treatment of Bloch's equations with isotropic diffusion terms (2) while Stejskal extended the treatment to include anisotropic diffusion (3), now commonly written for spatially uniform diffusion tensor as (Fig. 1)

$$
\frac{\partial \mathbf{M}}{\partial t}=\gamma \mathbf{M} \times \mathbf{B}-\frac{M_{x} \hat{\mathbf{x}}+M_{y} \hat{\mathbf{y}}}{T_{2}}-\frac{M_{z}-M_{0}}{T_{1}} \hat{\mathbf{z}}+\sum_{k=1}^{3} \sum_{l=1}^{3} \mathrm{D}_{k l} \partial_{k} \partial_{l} \mathbf{M}
$$

Here $\mathbf{M}$ is the magnetization vector; $t$ is time; $\gamma$ is the gyromagnetic ratio; $\mathbf{B}$ is the magnetic flux;

$\mathrm{T} 1$ and $\mathrm{T} 2$ are the longitudinal and transverse relaxation times, respectively; $\hat{\mathbf{x}}, \hat{\mathbf{y}}$ and $\hat{\mathbf{z}}$ are the three unit-axis of the Cartesian coordinates; $D_{k l}$ is the element of the diffusion tensor $\mathbf{D}$. The solution of Eq. [1] in the case of spin echo can be expressed as a linear relationship between the logarithm of the echo intensity and components of the diffusion tensor via (3) 


$$
\ln \left[\frac{s(\mathbf{b})}{s(0)}\right]=-\sum_{k=1}^{3} \sum_{l=1}^{3} b_{k l} D_{k l}=b_{k l} D_{k l}=\mathbf{b}: \mathbf{D},
$$

which forms the basis of diffusion tensor imaging (DTI) (4). Here, s(b) is the echo amplitude with diffusion weighting and $s(0)$ is that without diffusion weighting; $\mathbf{b}$ is the $\mathbf{b}$-matrix that describes the diffusion weighting in a pulse sequence.

Eq. [1\&2] work well if the underlying diffusion process is Gaussian. However, in most biological tissues such as the brain, the microstructural complexity renders the molecular motion to be non-Gaussian. To accommodate the non-Gaussian diffusion effect observed on diffusionweighted images of the brain, Liu et al. generalized Eq. [1] to include a series of higher-order tensors as follows (5-7)

$$
\begin{gathered}
\frac{\partial \mathbf{M}}{\partial t}=\gamma \mathbf{M} \times \mathbf{B}-\frac{M_{x} \hat{\mathbf{x}}+M_{y} \hat{\mathbf{y}}}{T_{2}}-\frac{M_{z}-M_{0}}{T_{1}} \hat{\mathbf{z}}+D_{i_{1} i_{2}}^{(2)} \partial_{i_{1}} \partial_{i_{2}} \mathbf{M}+D_{i_{1} i_{2} i_{3}}^{(3)} \partial_{i_{1}} \partial_{i_{2}} \partial_{i_{3}} \mathbf{M}+ \\
D_{i_{1} i_{2} i_{3} i_{4}}^{(4)} \partial_{i_{1}} \partial_{i_{2}} \partial_{i_{3}} \partial_{i_{4}} \mathbf{M}+\cdots
\end{gathered}
$$

Here, $D_{i_{1} i_{2} \ldots i_{n}}^{(n)}$ represents the elements of an $n$ th-order diffusion tensor. This generalization is made by generalizing Fick's first law to include higher order partial differentiations of spin concentrations. Eq. [3] can be solved analytically for the pulsed-gradient spin echo sequence, and the echo amplitude, similar to Eq. [2], is given by $(5,6)$

$$
\ln \left[\frac{s(\mathbf{b})}{s(0)}\right]=j^{2} b_{i_{1} i_{2}}^{(2)} D_{i_{1} i_{2}}^{(2)}+j^{3} b_{i_{1} i_{2} i_{3}}^{(3)} D_{i_{1} i_{2} i_{3}}^{(3)}+j^{4} b_{i_{1} i_{2} i_{3} i_{4}}^{(4)} D_{i_{1} i_{2} i_{3} i_{4}}^{(4)}+\cdots
$$

where $j$ is the imaginary unit and $b_{i_{1} i_{2} \ldots i_{n}}^{(n)}$ represents the elements of an $n$ th-order b-tensor determined by the amplitude and timing of the pulsed gradients. Eq. [4] shows that the oddordered diffusion tensors are encoded in the phase of signal while the even-ordered tensors are encoded in the magnitude. The series of higher-order tensors represents the covariance, 
skewness and kurtosis etc. (8). The relationship is best understood using a statistical analysis of the signal.

Eq. [4] represents a higher-order-cumulant expansion of the relationship between diffusion-weighted signal and the statistical properties of the underlying diffusion process following Eq. [3]. In practice, the infinite series sum has to be truncated. The fourth-order truncation of the higher order tensor model has been popularly referred as diffusion kurtosis imaging (DKI) (9). However, Eq. [4] does not necessarily fully characterize the complex diffusion environment of biological tissues, for example, the time-dependence of the cumulants is not considered, similar to DTI and DKI. A more in-depth discussion of the limitations can be found in the effective medium theory of a diffusion-weighted signal as described by Novikov and Kiselev (10).

\subsection{Statistical models}

While Bloch's equations with diffusion terms provide a phenomenological approach to quantify the diffusion signal with a set of diffusion tensors, the meanings of the tensors are not immediately clear. One way to investigate their meanings involves analyzing the diffusion signal using statistical approaches, in which the signal is treated as an ensemble average of all spins within a voxel (11) (Fig. 2). The signal originating from each spin is expressed as a complex number with a unit amplitude and a phase term that is path dependent. The phase of a given $\operatorname{spin} n$ ( $n=$ $1,2 \ldots N$ ) at time $t$ can be written as a discrete summation of path-dependent phase accruals as

$$
\Phi_{n}(t)=-\gamma \sum_{i=1}^{K} \boldsymbol{G}\left(t_{i}\right) \cdot \boldsymbol{r}_{n}\left(t_{i}\right) \delta t
$$

In generating the above summation, the time interval $t$ is discretized into $\mathrm{K}$ small intervals with $t_{i}$ representing the $i$-th interval. Each interval has a duration of $\delta t$. At a given time $t_{i}$, the position 
of the spin is given by $\boldsymbol{r}_{n}\left(t_{i}\right)$ where spin experiences a local field gradient vector of $\boldsymbol{G}\left(t_{i}\right)$. This treatment is generally referred to as the random model. The accuracy of the model can be made arbitrarily high by increasing $\mathrm{K}$, i.e. the number of time intervals.

For the pulsed gradient spin echo sequence, the summation of all spins gives the following echo amplitude

$$
S=\frac{1}{N} \sum_{n=1}^{N} e^{j\left(\Phi_{n}^{+}-\Phi_{n}^{-}\right)}=\frac{1}{N} \sum_{n=1}^{N} e^{j \Delta \Phi_{n}}
$$

where $\Phi_{n}^{+}$and $\Phi_{n}^{-}$represent the total phase accumulation of the $n$-th spin before and after the $180^{\circ}$-refocusing pulse, and $\Delta \Phi_{n}$ is the difference between the two. Under an ergodic assumption, the ensemble average in Eq. [6] can be replaced by the expected value of the $n$-th spin as follows

$$
s=\left\langle e^{j \Delta \Phi_{n}}\right\rangle=\left\langle e^{j \Delta \Phi}\right\rangle
$$

The subscript $n$ was dropped without loss of generality. Denote the sequence of random positions of a given spin as $\left\{r_{2 K} ; r_{2 K-1} \cdots r_{K+1} ; r_{K} \cdots r_{2} ; r_{1}\right\}$, where $r_{1}$ is the initial position, $\left\{r_{K} \cdots r_{2} ; r_{1}\right\}$ are the positions before the refocusing pulse and $\left\{r_{2 K} ; r_{2 K-1} \cdots r_{K+1}\right\}$ are the positions after the refocusing pulse. Further denote $p\left(r_{2 K} ; r_{2 K-1} \cdots r_{K+1} ; r_{K} \cdots r_{2} ; r_{1}\right)$ as the joint probability density function (PDF) for the sequence of random positions, then the echo amplitude can be evaluated as follows

$$
s=\int e^{j \Delta \Phi} p\left(r_{2 K} ; r_{2 K-1} \cdots r_{K+1} ; r_{K} \cdots r_{2} ; r_{1}\right) d r_{1} d r_{2} \cdots d r_{2 K}
$$

\subsubsection{Cumulant expansion}

Eq. [8] is essentially an integral over all particle trajectories-hence the phrase "path integral." This expression can be evaluated analytically only in some special cases. In one case (12), the transition probability between two adjacent positions is treated as independent and 
identically distributed (i.i.d.) Gaussian, in which case, a straightforward integration of Eq. [8] gives the exponential function shown in Eq. [2] where $D$ is proportional to the covariance matrix of the Gaussian PDF. In another case, if the diffusion-encoding gradient pulses are of infinitesimally narrow width and have infinitely high amplitude (i.e., a delta function), then Eq. [8] simplifies to the q-space formula as (13-15)

$$
s=\int e^{-j \gamma \delta \mathbf{G} \cdot \mathbf{r}} p(\mathbf{r}) d \mathbf{r}=\int e^{-j 2 \pi \mathbf{q} \cdot \mathbf{r}} p(\mathbf{r}) d \mathbf{r},
$$

where $p(r)$ denotes the probability distribution function (PDF) of net displacements, commonly referred to as the ensemble averaged propagator. For a general PDF, Eq. [9] can be expanded with a series of cumulant tensors (Fig. 3) which are shown to be proportional to the higher-order diffusion tensors $(5,6)$ in the case of pulsed-gradient spin echo (PGSE) sequence (Fig. 4). The underlying ensemble averaged propagator can be approximated with Gram-Charlier expansion using Hermite tensors $(5-7,16)$. A more general discussion of the cumulant expansion of diffusion signal can also be found in reference (17).

Eqs. [4\&9] both show that the phase of the diffusion signal is needed to reconstruct an asymmetrical PDF or asymmetrical underlying diffusion boundaries (Fig. 5). However, the symmetry of the pulsed gradient spin echo sequence suggests that its signal does not have a nontrivial phase term. In some situations, the symmetry can however be broken. For example, spin exchange in and out of an imaging voxel would break the symmetry and introduce phase in the diffusion signal $(5,6)$. The symmetry could also be broken with asymmetrical diffusion gradients. In one example, Laun et al. showed that the combination of long-narrow gradients was able to generate sufficient phase to reconstruct triangular-shaped pores $(18,19)$. 


\subsubsection{Mean apparent propagator (MAP) MRI}

An alternative representation of the MR signal attenuation is afforded by a series expansion of orthogonal basis functions. Similar to the cumulant expansion, this approach has its roots in statistics literature (20). Hermite functions, given by

$$
\phi_{n}(u, q)=\frac{j^{-n}}{\sqrt{2^{n} n !}} e^{-2 \pi^{2} q^{2} u^{2}} H_{n}(2 \pi u q) .
$$

are particularly advantageous in this endeavor (21) as the first basis function is a Gaussian whose width, determined by the parameter $u$, can be chosen to match the Gaussian employed in DTI while the others are orthogonal to it. Being the eigenfunctions of the Fourier operator, it is possible to represent both the signal and the propagator in the same basis. Note that the odd ordered terms are imaginary, thus accounting for any asymmetry of the propagator to be reconstructed. This representation has been found quite powerful as it is capable of representing even challenging signal profiles at a wide range of q-values (22).

The three-dimensional generalization of this approach has led to the development of the mean apparent propagator MRI (MAP-MRI) framework (23). A collection of scalar indices has been formulated based on this representation. The ability of the method to capture the higher q-behavior has enabled the estimation of the return-to-the origin probability (RTOP). A nonGaussianity metric has been formulated based on the dissimilarity of the real propagator to the Gaussian propagator, which is given simply by the first term in the series. The separability of the three-dimensional formulation into one-dimensional representations has been exploited to decompose the indices into orientations parallel and perpendicular to the principal diffusion orientation. The anisotropy of the propagator was also quantified, as well as the portion of anisotropy due solely to non-Gaussian terms. 
The efficiency provided by the Hermite function basis has enabled the implementation of the technique on data acquired in clinical scanners (24). Figure 6 illustrates the MAP-MRI indices as well as some common DTI metrics on data acquired in vivo using a clinical scanner.

\subsubsection{The confinement tensor model}

In a recent work, Yolcu et al. (25) introduced a new model of diffusion anisotropy based on a diffusive process involving particles subject to a spatially-linear (Hookean) restoring force (26). This problem was studied using three techniques; analytically via path integral methods (as discussed above for Gaussian diffusion), using a semi-analytic method via modifying the BlochTorrey equation, and numerically via random walk simulations; the results obtained were found to be in agreement.

In this model, the steady state distribution of particles is a Gaussian as illustrated in Figure 7 for the one-dimensional case. In three-dimensions, a tensorial force constant is employed, which leads to an oriented Gaussian distribution, end eventually, the anisotropy of the diffusion process (25). The problem has significant similarities with the much more challenging problem of restricted diffusion. In fact, there are several observations, which justify employing the confinement tensor model as a model of restricted diffusion. First, the mean-squared displacements in the confinement tensor framework, when plotted against the diffusion time, reaches a plateau just like in the case of restricted diffusion. This is a manifestation of the restricted diffusion, which justifies the employment of the model for representing the microdomains making up a compartmentalized specimen. Second, the convergence toward Gaussianity is indeed a well-known statistical characteristic of restricted diffusion; as the diffusion gradient pulse duration is prolonged, the distribution of the centers of mass for particle trajectories, and hence the MR signal approach a Gaussian (27), which is always the case for the 
confinement tensor model. Finally, the stochastic average effective force exerted by the walls diffusing in a restricted space has a linear dependence on the trajectories' center of mass at long durations. These observations have led to the identification of the confinement model as the "effective theory" of restricted diffusion for many acquisition scenarios (28).

\subsubsection{Tensor distribution models}

Yet another statistical approach involves considering the signal as the superposition of signal contributions originating from subdomains, each of which is represented by a diffusion tensor (29). Thus, the notion of a diffusion tensor distribution becomes relevant. In this picture, the detected signal is represented through a Laplace transform defined over the manifold of three-dimensional symmetric positive definite matrices $\mathcal{P}_{3}$ :

$$
S(\mathbf{b})=S_{0} \int_{\mathcal{P}_{3}} e^{-\operatorname{tr}(\mathbf{b} \mathbf{D})} \mathrm{d} F
$$

where $\mathrm{d} F$ is a probability measure, indicating the probability distribution of random diffusion tensors. Jian et al. (29) reported that when the ensemble of tensors is characterized by a central Wishart distribution, the signal expression can be evaluated analytically and is characterized by the expression

$$
S(\mathbf{b})=|\mathbf{I}+\mathbf{b} \mathbf{\Sigma}|^{-p},
$$

where $\mathbf{I}$ is the identity matrix, $p$ is the degree of freedom and $\boldsymbol{\Sigma}$ is the scale matrix of the Wishart distribution. The expected value of the tensor distribution is just $p \Sigma$. The above expression is the signal response for any gradient waveform. For the Stejskal-Tanner sequence, the signal is given by the Rigaut type asymptotic fractal expression. Remarkably, this expression had been successfully employed to represent the signal decay for various specimens (30). One important feature of such signal decay is its power-law tail (31), see Figure 8. In a recent work, the above 
findings were extended to non-central Wishart distributions; the generalized version was shown to yield improved accuracy when applied to the problem of resolving distinct fiber populations (32).

The tensor distribution models have been employed widely in recent years in studies aiming to elucidate the tissue microstructure. Such studies typically employ a model of microstructure, in which the tissue is thought to comprise distinct subdomains represented by a set of diffusion tensors, and the mesostructure is introduced via an orientation distribution function (33). Clearly, such models are special cases of the integral in Eq. [11]. The diffusion tensor distributions have also been employed in studies employing generalized gradient waveforms. For example, q-space trajectory imaging was introduced that employs acquisitions with measurement tensors of all ranks and it was shown that the second order mean diffusion tensor as well as the fourth order covariance tensor can be computed simply from the first and second terms of the Taylor series expansion of the signal (34). Several indices quantifying the various parameters of the microstructure have been introduced. In another interesting approach, Eq. 11 was reduced to a two-dimensional integral by taking or assuming the measurement and diffusion tensors to be axisymmetric (35). The simplified equation has been inverted in similar spirit with that in solid-state NMR techniques, yielding joint distributions of axial and radial diffusivities within heterogeneous specimens.

\subsection{Microstructural models}

Biological tissues are generally compartmentalized in part due to cell membranes that are semipermeable to water molecules. Given the limited spatial resolution of MRI, the diffusion processes within an imaging voxel are spatially heterogeneous. While statistical approaches for 
analyzing diffusion signals offer insights into the statistical properties of the diffusion process, inferring the microstructural components that give rise to the diffusion properties is not trivial. To address this limitation, various biophysical models have been developed by incorporating microstructural models of the underlying tissues (36-39). The general approach is to separate the tissue into multiple compartments with each compartment has distinctive diffusion properties, e.g. slow vs. faster diffusion, isotropic vs. anisotropic diffusion and unidirectional vs. dispersive diffusion. These compartments are generally derived based on the biological entities, e.g. in white matter, one may have myelin, axon and interstitial space. Details of these models are reviewed in other articles within this special issue.

\section{Integrating diffusion MRI with other modalities}

In this section, we review other imaging modalities as they relate to diffusion MRI. In many situations, these imaging techniques provide complementary information, thus enabling an improved delineation of the underlying tissue structure. For example, the capability of diffusion MRI in probing tissue microstructure is largely due to the existence of anisotropic diffusion arising from anisotropic tissue microstructures. These anisotropic tissue microstructures give rise not only to diffusion anisotropy but also other anisotropic properties such as structural tensor in anatomical images including light and electron microscopy, anisotropic relaxivities, internal magnetic field gradients and magnetic susceptibility anisotropy. Incorporating diffusion measurements into relaxation studies could provide more specificity on the distribution of microscopic compartments making up the complex specimen. As discussed below, diffusion MRI can also be employed as a functional imaging method with some potential for improving our ability to monitor physiological processes. 


\subsection{Magnetic susceptibility anisotropy and susceptibility tensor \\ 3.1.1 Susceptibility tensor imaging}

Recent studies have shown that magnetic susceptibility of biological tissues such as brain white matter $(40,41)$, myocardium $(42,43)$, kidney $(44)$ and cartilage $(45)$ is anisotropic, i.e. their susceptibility values depend on their orientations with respect to the $B_{0}$ field. These findings have led to the development of susceptibility tensor imaging which models the anisotropic magnetic susceptibility as a second-order tensor described as follows (40)

$$
f(\mathbf{k})=\boldsymbol{B}_{\mathbf{0}}\left(\frac{1}{3} \widehat{\mathbf{H}}^{T} \boldsymbol{\chi}(\mathbf{k}) \widehat{\mathbf{H}}-\widehat{\mathbf{H}} \cdot \mathbf{k} \frac{\mathbf{k}^{T} \boldsymbol{\chi}(\mathbf{k}) \widehat{\mathbf{H}}}{\boldsymbol{k}^{2}}\right) .
$$

Here, $f(\mathbf{k})$ is the Fourier transform of the frequency shift map; $\mathbf{k}$ is the $\mathbf{k}$-space vector; $\mathrm{B}_{0}$ is the strength of the applied magnetic flux density (field strength of the magnet); $\widehat{\mathbf{H}}$ is the unit vector of the applied magnetic field vector; $\chi(\mathbf{k})$ is the Fourier transform of the susceptibility tensor.

The susceptibility tensor shown in Eq. [13] is typically calculated from gradient echo phase images. Gradient echo phase can vary with respect to the relative orientation between the object (e.g. the brain) and the $B_{0}$ field simply due to the dipole field pattern; it can also vary due to anisotropic structural arrangement (46) and anisotropic magnetic susceptibility $(40,41)$. Estimating the susceptibility tensor using Eq. [13] requires a minimum of $6 \mathrm{~B}_{0}$ field orientations, which is typically accomplished by rotating the object within the magnet. As a result, in vivo studies utilizing STI have been limited due to the requirement to rotate the object.

\subsubsection{Susceptibility anisotropy vs. diffusion anisotropy in the brain}

In some cases, susceptibility anisotropy shares common microstructural components that underlie diffusion anisotropy; in many other cases, however, susceptibility and diffusion anisotropy have distinctive properties. Magnetic susceptibility anisotropy of brain white matter originates predominately from the myelin sheath while myelin accounts for less than $20 \%$ of 
diffusion anisotropy $(47,48)$. In a study of the dysmyelinating shiverer mouse that was genetically modified by deleting the genes encoding myelin basic protein, it was found that both the contrast between gray and white matter and the susceptibility anisotropy vanished, while diffusion anisotropy still appeared strong (47) (Fig. 9). Biophysical modeling also showed that specifically the radially aligned myelin lipids are the source of the orientation dependence of macroscopic magnetic susceptibility in normal mouse brain ex vivo and human brain in vivo (48). While both susceptibility and diffusion generally show high anisotropy in the white matter, clear differences have also been reported. For example, Li et al. showed that there is a lack of correlation between susceptibility anisotropy and diffusion anisotropy in human brain white matter (49). In major white matter tracts, while STI-reconstructed fibers appeared similar to DTI-reconstructed fibers, there was clear divergence in fiber orientations and sensitivity (50) (Fig. 10a). The performance of STI and DTI fiber tracking has also been compared in the myocardium of mouse heart (42,43)(Fig. 10b).

\subsubsection{Susceptibility anisotropy vs. diffusion anisotropy in the kidney}

Xie et al. investigated the susceptibility and diffusion anisotropy of renal tubules in the mouse kidney (44). In this study, STI data were acquired using a gradient multi-echo sequence on perfusion fixed mouse kidney on a 9.4T scanner. Apparent magnetic susceptibility demonstrated an orientation dependence of susceptibility contrast in the tubules. One surprising finding was that, STI was able to detect tracks in the renal cortex and outer medullar where DTI failed (Fig. 10c). The study also found that STI eigenvector pointing along the tubule axis was the minor eigenvector instead of the major eigenvector as occurred in the brain, suggesting that there is a consistent diamagnetic content in the direction of the renal tubule long axis. The renal epithelial cells generally have large surface area - this is critical for fluid absorption and for increasing the 
number of membrane proteins in the kidney as well as other organs. The surface amplification is achieved through densely arranged microvilli of the brush border on the luminal (apical) plasma membrane and basement infoldings on the basolateral membrane. It was reasoned that these structures significantly increase the percentage of lipids pointing along the tubule's long axis which gives rise to the observed susceptibility anisotropy. The reasons that DTI failed to reconstruct the tubules in the cortex and outer medullar are the short diffusion distance compared to the diameters of the tubules and the tortuosity of the tubules. For example, the expected diffusion displacement measured in the experiments was $5.2 \mu \mathrm{m}$, while in the outer medullar, the thin limbs had limited straight segments, and the tubules also had larger diameters up to $46.7 \mu \mathrm{m}$. As a result, water diffusion would appear to be isotropic, and DTI was not successful in representing the anisotropic structure in the outer medullar.

\subsubsection{Susceptibility anisotropy vs. diffusion anisotropy in the cartilage}

In certain cases, susceptibility anisotropy is demonstratively more sensitive to tissue microstructure than diffusion anisotropy. One recent study showed that susceptibility anisotropy within articular cartilage produces zone-specific magnetic susceptibility contrast (45). When collagen fibrils are mostly oriented perpendicular to B0 field, the susceptibility values are more paramagnetic, e.g. in the superficial zone. When collagen fibrils are mostly parallel to B0 field, the susceptibility values become more diamagnetic, e.g. in the deep zone. The macroscopic susceptibility anisotropy of collagen was believed to originate from the diamagnetic anisotropy of the peptide group at the molecular level $(45,51)$. The collagen triple helix contains peptide group planes oriented at approximately $45^{\circ}$ to the fibril axis. Because the most diamagnetic susceptibility is oriented normal to the peptide group plane, the net susceptibility of collagen fibrils is mostly diamagnetic in the direction parallel to the fibril axis. Cumulatively, these 
organized peptide groups yield the greatest diamagnetism in the direction of the collagen molecule long axis. This trend is the opposite of the susceptibility anisotropy observed in brain white matter. Tractography results revealed that STI clearly depicted the collagen fibril orientation beyond the capability of diffusion tensor imaging (DTI) (51) (Fig. 10d).

\subsection{Structure tensor}

Structure tensor is a quantity used commonly in image texture analysis. Structure tensor $\mathbf{S}_{w}(\mathbf{r})$ of a $2 \mathrm{D}$ image I (or a function in general) is a matrix defined based on the spatial gradients of the image as follows (52)

$$
\begin{gathered}
S_{w}(\mathbf{r})=w(\mathbf{r}) * S_{0}(\mathbf{r}) \\
S_{0}(\mathbf{r})=\left[\begin{array}{ll}
I_{x}(\mathbf{r}) I_{x}(\mathbf{r}) & I_{x}(\mathbf{r}) I_{y}(\mathbf{r}) \\
I_{x}(\mathbf{r}) I_{y}(\mathbf{r}) & I_{y}(\mathbf{r}) I_{y}(\mathbf{r})
\end{array}\right]
\end{gathered}
$$

where " $*$ " represents the convolution operation and $w(\mathbf{r})$ is a weighting function (e.g. Gaussian). Structure tensor can be used to identify the predominant directions of the gradient in a specified neighborhood of a voxel, and the level to which those directions are coherent. This can be achieved by eigenvalue decomposition of the structure tensor to derive local orientation, anisotropy, and intensity similar to what's done in DTI. Some recent studies have used structure tensor analysis of histological slides as a means to validate DTI. In one study, anisotropy measured with structure tensor analysis of stained histological sections was found to be highly correlated with anisotropy measured by ex vivo DTI of the same brains $\left(R^{2}=0.92\right)(53)$. The same group also applied the technique to study the pathological substrates underlying the significant DTI abnormalities following traumatic brain injury (54). Using a rat model of controlled cortical impact, the study attempted to identify the specific pathological features that give rise to the diffusion tensor imaging changes in subacute to chronic traumatic brain injury. Cortical anisotropy was found to be independently associated (standardized $\beta=0.62, P=0.04$ ) with the coherent organization of reactive astrocytes (i.e. gliosis) and was not attributed to axons. On the other hand, it was 
reported that a decrease in white matter anisotropy $(P<0.001)$ was significantly related to demyelination $(\beta=0.75, P=0.0015)$ and to a lesser extent, axonal degeneration $(\beta=-0.48, P=0.043)$. Gliosis within the lesioned cortex was also found to influence diffusion tensor tractography; it was argued that spurious tracts observed in the injured brain might not necessarily reflect continuous axons and might instead depict glial scarring.

Structure tensor has also been used to inform diffusion-based fiber tractography by combining diffusion-weighted images and gradient-echo images (55). This structure tensor informed fiber tractography (STIFT) takes advantage of the white matter and gray matter contrast shown in gradient echo images at high field. The study described a method to calculate a data-adaptive structure tensor from the gradient echo image to describe the morphology of fiber bundles. The structure tensor was then incorporated in a tractography algorithm to modify the diffusion-based tracking direction according to structure tensor properties. The study reported that this gradient-echo structure tensor was informative for tractography. In one example, reconstruction of the optic radiation with STIFT showed a larger anterior extent of Meyer's loop compared to a standard tractography alternative. In another example, STIFT in multifiber voxels yielded a reduction in crossing-over of streamlines from the cingulum to the adjacent corpus callosum, while tracking through the fiber crossings of the centrum semiovale was unaffected (55). More studies are needed to verify the improvements reported by STIFT.

\subsection{Internal magnetic field gradient}

While the pulsed-gradient spin echo sequence uses strong gradient pulses to encode diffusion and reduces the relative contributions from the internal gradients, one may also use the diffusion effect in the internal gradient field to infer microstructural properties. In highly porous structures such as the trabecular bone system, the largest internal gradients (and thus regions of fast signal decay) exist close to the trabecular surfaces that are perpendicular to the applied field. In one study, signal decay due to diffusion in the internal field (DDIF) contrast was investigated in two different water saturated bovine 
trabecular bone samples (56). The results confirmed that the DDIF contrast is related to the surface-tovolume ratio. A clear correlation was reported between the maxima in the DDIF rate and the minima in the spin density at the trabecular rod/plate locations. The distributions of DDIF rates were reportedly to qualitatively mimic the shapes seen in the bulk sample results, but show a slight dependence on image slice orientation, which the authors attributed to variation in cross-term diffusion weighting between internal and externally applied field gradients. The study concluded that although DDIF may potentially be less sensitive, it may offer a more specific contrast for trabecular number tracking than the $T_{2}{ }^{\prime}$ approach. In another example, the diffusion in the internal field was used to visualize internal magnetic field gradients arising from susceptibility contrast between an array of cylindrical glass tubes (solid matrix) and surrounding water (pore fluids) in a uniform applied magnetic field (57). A stimulated echo sequence was performed to image the decay rates due to diffusion in the internal magnetic field. The authors computed the interference pattern of the cross-terms between the internal and the applied field gradient and used that to extract the orientation of the internal field gradient. The experimental results were found to be consistent with the theoretical calculations.

Without relying on diffusion effect, the internal field gradients may also be mapped using gradient echo frequency shift. In the zeroth order approximation, the mean magnetic susceptibility or susceptibility tensor can be measured using quantitative susceptibility mapping (QSM) (58-63) or susceptibility tensor imaging (40) respectively. QSM and STI estimates the mean magnetic susceptibility of a whole voxel. To obtain higher-order information, specifically the distribution of magnetic field within a voxel, Liu and Li proposed to apply an external field gradient, before the gradient echo readout, which will interfere with the internal field gradient and affect the mean frequency shift of the voxel (64). Since the amplitude and direction of the external gradient can be controlled, this interference has been shown to be able to estimate the distribution of the internal field in some simulated cases and areas of mouse brains. However, 
the contrast induced by the gradient interference is generally weaker than the zeroth order information, thus difficult to estimate accurately.

\subsection{Diffusion-Relaxation Correlation}

Heterogeneous specimens are often characterized by a distribution of MR related parameters. For example, different transverse relaxation rates can be associated with different compartments within the medium. Let $R_{2}=1 / T_{2}$ denote the transverse relaxivity and $p\left(R_{2}\right)$ be the distribution of relaxivities. The MR signal collected at the echo time TE can be written through the expression

$$
S(T E)=\int_{0}^{\infty} p\left(R_{2}\right) e^{-T E R_{2}} \mathrm{~d} R_{2}
$$

In other words, the signal is simply the Laplace transform of the relaxivity distribution. Thus, repeating the acquisition with a number of echo times, and inverting the Laplace transform would provide an estimate of $p\left(R_{2}\right)$.

The same idea can be employed for the diffusive characteristics, which is often reduced to a scalar-valued diffusion coefficient for each compartment. The experimentally controlled parameter is the b-value, and the analogous relationship is

$$
S(b)=\int_{0}^{\infty} p(D) e^{-b D} \mathrm{~d} D
$$

In many situations of practical interest, distribution of relaxation times or diffusion coefficients alone is not sufficiently descriptive of the specimen's structure. Probing the distribution of both characteristics would make it possible to distinguish separate compartments that share the same relaxivity or diffusivity value. Multi-dimensional techniques have been introduced precisely for this purpose. From a practical point of view, a two-dimensional D-TE "plane" is sampled at a large number of points, and a joint distribution $p\left(D, R_{2}\right)$ is computed by inverting the two-dimensional Laplace transform $(65,66)$

$$
S(b, T E)=\int_{0}^{\infty} \int_{0}^{\infty} p\left(D, R_{2}\right) e^{-T E R_{2}} e^{-b D} \mathrm{~d} R_{2} \mathrm{~d} D
$$


Note that $p(D)$ and $p(R 2)$ are the one-dimensional projections of the joint density $p(R 2, D)$. In NMR spectroscopy literature, this two-dimensional technique is referred to as diffusion-relaxation correlation spectroscopy (DR-COSY). Inverting the Laplace transform, whether one- or multi-dimensional is a challenging but necessary component of all these methods, and several algorithms have been developed in that literature over the years to address this important issue $(67,68)$. Very recently, a novel pulse sequence featuring frequency-swept RF pulses along with gradients was devised that introduces different amounts of diffusion weightings at different $z$ coordinates within the specimen (69). Thus, a onedimensional "image" provides the entire set of data points along the b-axis. When this sequence is followed by a Carr-Purcell-Meiboom-Gill (CPMG) pulse train, the entire b-TE plane can, in principle, be sampled in one-shot, providing one to two orders of magnitude gain in total acquisition time.

The above-mentioned challenges are invariably intensified when one attempts to spatially localize the NMR signal as in imaging studies (70). Ever increasing demand for accurately characterizing tissue structure alongside technological advances that has improved data quality in clinical scanners has prompted some groups to incorporate two-dimensional relaxation-diffusion encoding into standard image acquisitions for biomedical applications (71). To overcome the said difficulties, Kim et al. (72) utilized a spatial regularization scheme in addition to the commonly used non-negativity constraint and employed their method on a mouse model of traumatic spinal cord injury. Benjamini and Basser (73) introduced physical constrains to the problem based on the one-dimensional marginal distributions; they report a substantial reduction in the required number of data points thereby shortening the acquisition time. In a recent study (74), the same authors reported that the two-dimensional spectra revealed different tissue components such as axons, neuronal and glial soma, macromolecules within myelin, and interstitial space in ferret spinal cord. 


\subsection{Diffusion-based fMRI}

Over the last three decades, there have been a number of attempts in employing the diffusion-attenuated MR signal as a marker for brain activation. The progress over the years has closely followed technological advances, most notably by introducing larger and larger diffusion weightings while maintaining an acceptable signal to noise ratio.

Le Bihan et al. (75) argued that DWIs at b-values less than $100 \mathrm{~s} / \mathrm{mm}^{2}$ would be sensitive to blood microcirculation linked to a hemodynamic response, like in $\mathrm{T}^{*}$-weighted blood oxygenation level dependent (BOLD) functional MRI (fMRI) (76-78). However, the latter offered better sensitivity and reliability. Zhong et al. reported increased apparent diffusion coefficient $(A D C)$ values due to visual stimulation at higher $b$-values $\left(700 \mathrm{~s} / \mathrm{mm}^{2}\right)$ attributing the effect to changes in local magnetic field gradients (79). An opposite effect, i.e., a slight reduction in the ADC values, was observed at b-values of around $1500 \mathrm{~s} / \mathrm{mm}^{2}(80)$. The underlying factor was speculated to be not related to blood flow, but due to cortical cells undergoing a transient swelling.

The question of employing diffusion MRI as a functional imaging technique was revisited by performing measurements at even higher $b$-values (up to $b=2400 \mathrm{~s} / \mathrm{mm}^{2}$ ) (81). In this $b$-value range, a single signal decay rate (diffusion coefficient) is insufficient to represent the signal profile. Instead, the biexponential model, given by

$$
S=S_{0} f e^{-b D_{\text {slow }}}+S_{0}(1-f) e^{-b D_{\text {fast }}},
$$

was used, which enables one to compute two diffusion coefficients. Here, $f$ represents the signal fraction corresponding to the slower diffusion component, i.e., the one with smaller diffusivity. As shown in Figure 11 (left), as more and more diffusion-weighting is introduced, the signal 
increase is observed 2-3 seconds earlier than that in the BOLD fMRI response. The signal profiles appear qualitatively similar, though the initial signal undershoot is absent in the case of diffusionweighted signals. Moreover, the percentage signal increase is comparable to that in BOLD fMRI at larger b-values. The underlying mechanism of such changes have been proposed to be cell swelling, which is thought to alter the membrane surface area, thus changing the fraction of molecules bound to the membranes. These findings have prompted a number of studies aiming to reproduce the results and assess the possible underlying mechanisms.

Tsurugizawa et al. (82) reported that employing nitroprusside (a neurovascular coupling inhibitor) removed the hemodynamic response as expected. However, the local field potentials and diffusion response were maintained for the most part, suggesting that the underlying mechanisms of the signal changes observed via BOLD fMRI and diffusion fMRI could be different. More recent findings (83) based on ethanol injection into rat brains suggest that in the presence of complications due to vasoactive drugs, the diffusion fMRI signal outperforms the BOLD fMRI signal as an indicator of neuronal activity.

Miller et al. (84) performed measurements of mild hypercapnia, which alters the blood flow without affecting neuronal firing. Their findings suggested a signal change, which is similar to the trend in diffusion fMRI signal profile, i.e., the signal increases as b-value is increased. Moreover, the diffusion fMRI response did not appear to be substantially earlier than the $2^{*}$ weighted BOLD response. These observations lead to the conclusion that a substantial part of the diffusion fMRI signal change could be of vascular origin. A similar conclusion was reached in a recent study (85) performed on organotypic cortex cultures examined simultaneously via calcium fluorescence imaging and diffusion MR acquisitions performed using a one-sided desktop MR 
system (86). Using tissue cultures is advantageous in that neuroactivity can be manipulated and monitored without the confounding effects such as hemodynamic changes. The authors observed an increase in diffusion-weighted signal coupled to the prolonged depolarization of neurons. However, when neuronal activity is suppressed, e.g., via introducing tetrodotoxin, the diffusion-weighted MR signal and the signal fraction for slow diffusion did not appear to change

(Fig. 12) suggesting the lack of correlation with spontaneous neuronal activity.

The above findings are largely contradictory especially with regards to the timing of the diffusion-weighted signal change and also to its biophysical underpinnings. It should be noted however, that the biological models, as well as the experimental framework to investigate them, substantially differ in these studies. For reliably utilizing diffusion MRI as a functional imaging technique, there appears to be a need for establishing its feasibility and sensitivity in various situations. Poorer signal to noise ratio afforded by high b-value diffusion weighted acquisitions calls for the development of a robust and reliable diffusion $\mathrm{fMRI}$ protocol as well as an optimized data processing pipeline for clinical studies (87). Last but not least, a correct interpretation of the results would be achieved after a better understanding of the mechanisms that lead to the observed signal changes.

\section{Discussions and Conclusions}

Molecular diffusion has become one of the most widely used contrast in MRI, both for clinical applications and basic research. While the fundamental principle, i.e., random motion in inhomogeneous field inducing phase dispersion, has been understood since the early days of NMR, the understanding of diffusion signal measured by MRI in biological tissues, especially the brain, remains incomplete. The complexity of this relationship is a result of the complexity of 
physiology and the extreme sensitivity of diffusion to tissue cellular structure. The relationship between diffusion MRI signal and tissue structure and function remains a highly active area of research. Integrating other information provided by MR or non-MR imaging modalities might be a key to improve our understanding of how diffusion MRI relates to physiology and biology.

\section{Acknowledgement}

EÖ acknowledges support by Linköping University Center for Industrial Information Technology (CENIIT), the Swedish Foundation for Strategic Research AM13-0090 and the Swedish Research Council 2015-05356 and 2016-04482. 


\section{Reference:}

1. Hahn EL. Spin echoes. Phys. Rev. 1950;80:580-594. doi: 10.1103/PhysRev.80.580.

2. Torrey HC. Bloch equations with diffusion terms. Phys. Rev. 1956;104:563-565. doi: 10.1103/PhysRev.104.563.

3. Stejskal EO. Use of Spin Echoes in a Pulsed Magnetic-Field Gradient to Study Anisotropic, Restricted Diffusion and Flow. J. Chem. Phys. [Internet] 1965;43:3597-3603. doi:

10.1063/1.1696526.

4. Basser PJ, Mattiello J, LeBihan D. MR diffusion tensor spectroscopy and imaging. Biophys J [Internet] 1994;66:259-267. doi: S0006-3495(94)80775-1 [pii] \r10.1016/S0006-3495(94)807751.

5. Liu C, Bammer R, Moseley ME. Generalized Diffusion Tensor Imaging (GDTI): A Method for Characterizing and Imaging Diffusion Anisotropy Caused by Non-Gaussian Diffusion. Isr. J. Chem. [Internet] 2003;43:145-154. doi: 10.1560/HB5H-6XBR-1AW1-LNX9.

6. Liu C, Bammer R, Acar B, Moseley ME. Characterizing Non-Gaussian Diffusion by Using Generalized Diffusion Tensors. Magn. Reson. Med. 2004;51:924-937. doi: 10.1002/mrm.20071.

7. Liu C, Bammer R, Acar B, Moseley ME. Generalized diffusion tensor imaging (GDTI) using higher-order tensor (HOT) statistics. In: Proceedings of the 11th ISMRM. Toronto; 2003. p. 242.

8. Stepišnik J. Analysis of NMR self-diffusion measurements by a density matrix calculation. Phys. B+C 1981;104:350-364. doi: 10.1016/0378-4363(81)90182-0.

9. Jensen JH, Helpern JA, Ramani A, Lu H, Kaczynski K. Diffusional kurtosis imaging: The quantification of non-Gaussian water diffusion by means of magnetic resonance imaging. Magn. Reson. Med. 2005;53:1432-1440. doi: 10.1002/mrm.20508.

10. Novikov DS, Kiselev VG. Effective medium theory of a diffusion-weighted signal. NMR Biomed. 2010;23:682-697. doi: 10.1002/nbm.1584.

11. Torrey HC. Nuclear spin relaxation by translational diffusion. Phys. Rev. 1953;92:962-969. doi: 10.1103/PhysRev.92.962.

12. Özarslan E, Westin CF, Mareci TH. Characterizing magnetic resonance signal decay due to gaussian diffusion: The path integral approach and a convenient computational method.

Concepts Magn. Reson. Part A Bridg. Educ. Res. 2015;44:203-213. doi: 10.1002/cmr.a.21354.

13. Callaghan PT. Pulsed field gradient nuclear magnetic resonance as a probe of liquid state molecular organization. Aust. J. Phys. 1984;37:359-387.

14. Callaghan PT. PGSE-MASSEY, a sequence for overcoming phase instability in very-highgradient spin-echo NMR. J. Magn. Reson. 1990;88:493-500. doi: 10.1016/0022-2364(90)90283F. 
15. Callaghan PT, Coy A, MacGowan D, Packer KJ, Zelaya FO. Diffraction-like effects in NMR diffusion studies of fluids in prous solids. Nature 1991;351:467-469.

16. Liu C, Mang SC, Moseley ME. In vivo generalized diffusion tensor imaging (GDTI) using higher-order tensors (HOT). Magn. Reson. Med. 2010;63:243-252. doi: 10.1002/mrm.22192.

17. Kiselev VG. Chapter 10: The Cumulant Expansion: An Overarching Mathematical Framework For Understanding Diffusion NMR. In: Jones DK, editor. Diffusion MRI Theory, Methods, and Applications. New York: Oxford University Press; 2010. pp. 152-168.

18. Laun FB, Kuder TA, Semmler W, Stieltjes B. Determination of the defining boundary in nuclear magnetic resonance diffusion experiments. Phys. Rev. Lett. 2011;107. doi: 10.1103/PhysRevLett.107.048102.

19. Laun FB, Kuder TA, Wetscherek A, Stieltjes B, Semmler W. NMR-based diffusion pore imaging. Phys. Rev. E - Stat. Nonlinear, Soft Matter Phys. 2012;86. doi: 10.1103/PhysRevE.86.021906.

20. Schwartz SC. Estimation of Probability Density by an Orthogonal Series. Ann. Math. Stat. [Internet] 1967;38:1261-1265. doi: 10.1214/aoms/1177698795.

21. Ozarslan E, Koay C, Basser P. Simple harmonic oscillator based estimation and reconstruction for one-dimensional q-space MR. In: Proceedings 16th Scientific Meeting, International Society for Magnetic Resonance in Medicine. Toronto, Ontario, Canada; 2008. p. 35 .

22. Özarslan E, Shemesh N, Koay CG, Cohen Y, Basser PJ. Nuclear magnetic resonance characterization of general compartment size distributions. New J. Phys. 2011;13. doi: 10.1088/1367-2630/13/1/015010.

23. Özarslan E, Koay CG, Shepherd TM, Komlosh ME, Irfanoğlu MO, Pierpaoli C, Basser PJ. Mean apparent propagator (MAP) MRI: A novel diffusion imaging method for mapping tissue microstructure. Neuroimage 2013;78:16-32. doi: 10.1016/j.neuroimage.2013.04.016.

24. Avram A V., Sarlls JE, Barnett AS, Ozarslan E, Thomas C, Irfanoglu MO, Hutchinson E, Pierpaoli C, Basser PJ. Clinical feasibility of using mean apparent propagator (MAP) MRI to characterize brain tissue microstructure. Neuroimage 2016;127:422-434. doi: 10.1016/j.neuroimage.2015.11.027.

25. Yolcu C, Memiç M, Şimşek K, Westin C, Özarslan E. NMR signal for particles diffusing under potentials: From path integrals and numerical methods to a model of diffusion anisotropy. Phys. Rev. E - Stat. Nonlinear, Soft Matter Phys. 2016;93:52602. doi: https://doi.org/10.1103/PhysRevE.93.052602.

26. Uhlenbeck GE, Ornstein LS. On the theory of the Brownian motion. Phys. Rev. 1930;36:823841. doi: 10.1103/PhysRev.36.823.

27. Mitra PP, Halperin BI. Effects of Finite Gradient-Pulse Widths in Pulsed-Field-Gradient Diffusion Measurements. J. Magn. Reson. Ser. A 1995;113:94-101. doi: 
10.1006/jmra.1995.1060.

28. Özarslan E, Yolcu C, Herberthson M, Westin CF, Knutsson H. Effective potential for magnetic resonance measurements of restricted diffusion. Front. Phys. 2017;5:68.

29. Jian B, Vemuri BC, Özarslan E, Carney PR, Mareci TH. A novel tensor distribution model for the diffusion-weighted MR signal. Neuroimage 2007;37:164-176. doi:

10.1016/j.neuroimage.2007.03.074.

30. Köpf M, Metzler R, Haferkamp O, Nonnenmacher TF. NMR studies of anomalous diffusion in biological tissues: experimental observation of Lévy stable processes. In: Losa GA, Merlini D, Nonnenmacher TF, Weibel ER, editors. Fractals in Biology and Medicine, Vol. 2. Birkhäuser, Basel; 1998. pp. 354-364.

31. Sen PN, Hürlimann MD, De Swiet TM. Debye-Porod law of diffraction for diffusion in porous media. Phys. Rev. B 1995;51:601-604. doi: 10.1103/PhysRevB.51.601.

32. Shakya S, Batool N, Özarslan E, Knutsson H. Multi-fiber reconstruction using probabilistic mixture models for diffusion MRI examinations of the brain. In: Hotz I, Özarslan E, Schultz T, editors. Modeling, Analysis, and Visualization of Anisotropy. Cham: Springer International Publishing; 2017. pp. 283-308.

33. Reisert M, Kellner E, Dhital B, Hennig J, Kiselev VG. Disentangling micro from mesostructure by diffusion MRI: A Bayesian approach. Neuroimage 2017;147:964-975. doi: 10.1016/j.neuroimage.2016.09.058.

34. Westin CF, Knutsson $\mathrm{H}$, Pasternak O, et al. Q-space trajectory imaging for multidimensional diffusion MRI of the human brain. Neuroimage 2016;135:345-362. doi:

10.1016/j.neuroimage.2016.02.039.

35. De Almeida Martins JP, Topgaard D. Two-Dimensional Correlation of Isotropic and Directional Diffusion Using NMR. Phys. Rev. Lett. 2016;116. doi:

10.1103/PhysRevLett.116.087601.

36. Zhang H, Schneider T, Wheeler-Kingshott CA, Alexander DC. NODDI: Practical in vivo neurite orientation dispersion and density imaging of the human brain. Neuroimage 2012;61:10001016. doi: 10.1016/j.neuroimage.2012.03.072.

37. Assaf $Y$, Basser PJ. Composite hindered and restricted model of diffusion (CHARMED) MR imaging of the human brain. Neuroimage 2005;27:48-58. doi:

10.1016/j.neuroimage.2005.03.042.

38. Assaf Y, Blumenfeld-Katzir T, Yovel Y, Basser PJ. AxCaliber: A method for measuring axon diameter distribution from diffusion MRI. Magn. Reson. Med. 2008;59:1347-1354. doi: 10.1002/mrm.21577.

39. Fieremans $\mathrm{E}$, Jensen $\mathrm{JH}$, Helpern JA. White matter characterization with diffusional kurtosis imaging. Neuroimage 2011;58:177-188. doi: 10.1016/j.neuroimage.2011.06.006.

40. Liu C. Susceptibility tensor imaging. Magn. Reson. Med. 2010;63:1471-1477. doi: 
10.1002/mrm.22482.

41. Lee J, Shmueli K, Fukunaga M, van Gelderen P, Merkle H, Silva AC, Duyn JH. Sensitivity of MRI resonance frequency to the orientation of brain tissue microstructure. Proc. Natl. Acad. Sci. USA [Internet] 2010;107:5130-5135. doi: 10.1073/pnas.0910222107.

42. Dibb R, Liu C. Joint eigenvector estimation from mutually anisotropic tensors improves susceptibility tensor imaging of the brain, kidney, and heart. Magn. Reson. Med. 2016;0:1-16. doi: $10.1002 / \mathrm{mrm} .26321$.

43. Dibb R, Qi Y, Liu C. Magnetic susceptibility anisotropy of myocardium imaged by cardiovascular magnetic resonance reflects the anisotropy of myocardial filament $\alpha$-helix polypeptide bonds. J. Cardiovasc. Magn. Reson. [Internet] 2015;17:60. doi: 10.1186/s12968015-0159-4.

44. Xie L, Dibb R, Cofer GP, Li W, Nicholls PJ, Johnson GA, Liu C. Susceptibility tensor imaging of the kidney and its microstructural underpinnings. Magn. Reson. Med. 2015;73:1270-1281. doi: $10.1002 / \mathrm{mrm} .25219$.

45. Wei H, Dibb R, Decker K, Wang N, Zhang Y, Zong X, Lin W, Nissman DB, Liu C. Investigating magnetic susceptibility of human knee joint at 7 tesla. Magn. Reson. Med. [Internet] 2017;0:111. doi: 10.1002/mrm.26596.

46. He X, Yablonskiy D a. Biophysical mechanisms of phase contrast in gradient echo MRI. Proc. Natl. Acad. Sci. U. S. A. [Internet] 2009;106:13558-13563. doi: 10.1073/pnas.0904899106.

47. Liu C, Li W, Johnson GA, Wu B. High-field (9.4T) MRI of brain dysmyelination by quantitative mapping of magnetic susceptibility. Neuroimage 2011. doi: 10.1016/j.neuroimage.2011.02.024.

48. Li W, Wu B, Avram A V., Liu C. Magnetic susceptibility anisotropy of human brain in vivo and its molecular underpinnings. Neuroimage [Internet] 2012;59:2088-2097. doi:

10.1016/j.neuroimage.2011.10.038.

49. Li X, Vikram DS, Lim IAL, Jones CK, Farrell JAD, van Zijl PCM. Mapping magnetic susceptibility anisotropies of white matter in vivo in the human brain at 7T. Neuroimage 2012;62:314-330. doi: 10.1016/j.neuroimage.2012.04.042.

50. Li W, Liu C. Comparison of Magnetic Susceptibility Tensor and Diffusion Tensor of the Brain. J. Neurosci. neuroengineering [Internet] 2013;2:431-440. doi: 10.1166/jnsne.2013.1075.

51. Wei H, Gibbs E, Zhao P, Wang N, Cofer GP, Zhang Y, Johnson GA, Liu C. Susceptibility tensor imaging and tractography of collagen fibrils in the articular cartilage. Magn. Reson. Med. 2017;78. doi: 10.1002/mrm.26882.

52. Bigun J, Granlund GH. Optimal Orientation Detection of Linear Symmetry. Proc. IEEE First Int. Conf. Comput. Vis. 1987;54:433-438. doi: 10.1.1.429.961.

53. Budde MD, Frank JA. Examining brain microstructure using structure tensor analysis of histological sections. Neuroimage 2012;63:1-10. doi: 10.1016/j.neuroimage.2012.06.042. 
54. Budde MD, Janes L, Gold E, Turtzo LC, Frank JA. The contribution of gliosis to diffusion tensor anisotropy and tractography following traumatic brain injury: Validation in the rat using Fourier analysis of stained tissue sections. Brain 2011;134:2248-2260. doi: 10.1093/brain/awr161.

55. Kleinnijenhuis $M$, Barth M, Alexander DC, van Cappellen van Walsum AM, Norris DG. Structure Tensor Informed Fiber Tractography (STIFT) by combining gradient echo MRI and diffusion weighted imaging. Neuroimage 2012;59:3941-3954. doi: 10.1016/j.neuroimage.2011.10.078.

56. Sigmund $\mathrm{EE}$, Cho $\mathrm{H}$, Song YQ. High-resolution MRI of internal field diffusion-weighting in trabecular bone. NMR Biomed. 2009;22:436-448. doi: 10.1002/nbm.1354.

57. Cho H, Ryu S, Ackerman JL, Song Y-Q. Visualization of inhomogeneous local magnetic field gradient due to susceptibility contrast. J. Magn. Reson. [Internet] 2009;198:88-93. doi: 10.1016/j.jmr.2009.01.024.

58. Li J, Chang S, Liu T, et al. Reducing the object orientation dependence of susceptibility effects in gradient echo MRI through quantitative susceptibility mapping. Magn. Reson. Med. 2012;68:1563-1569. doi: 10.1002/mrm.24135.

59. Li W, Wu B, Liu C. Quantitative susceptibility mapping of human brain reflects spatial variation in tissue composition. Neuroimage 2011;55:1645-1656. doi: 10.1016/j.neuroimage.2010.11.088.

60. Shmueli K, de Zwart JA, van Gelderen P, Li T-Q, Dodd SJ, Duyn JH. Magnetic susceptibility mapping of brain tissue in vivo using MRI phase data. Magn. Reson. Med. [Internet] 2009;62:1510-22. doi: 10.1002/mrm.22135.

61. Wang Y, Liu T. Quantitative susceptibility mapping (QSM): Decoding MRI data for a tissue magnetic biomarker. Magn. Reson. Med. 2015;73:82-101. doi: 10.1002/mrm.25358.

62. Li W, Wang N, Yu F, Han H, Cao W, Romero R, Tantiwongkosi B, Duong TQ, Liu C. A method for estimating and removing streaking artifacts in quantitative susceptibility mapping. Neuroimage 2015. doi: 10.1016/j.neuroimage.2014.12.043.

63. Liu C, Li W, Tong KA, Yeom KW, Kuzminski S. Susceptibility-weighted imaging and quantitative susceptibility mapping in the brain. J. Magn. Reson. Imaging 2015;42:23-41. doi: 10.1002/jmri.24768.

64. Liu C, Li W. Imaging neural architecture of the brain based on its multipole magnetic response. Neuroimage [Internet] 2013;67:193-202. doi: 10.1016/j.neuroimage.2012.10.050.

65. Callaghan PT, Godefroy S, Ryland BN. Use of the second dimension in PGSE NMR studies of porous media. In: Magnetic Resonance Imaging. Vol. 21. ; 2003. pp. 243-248. doi: 10.1016/S0730-725X(03)00131-0.

66. Hürlimann MD, Venkataramanan L. Quantitative measurement of two-dimensional distribution functions of diffusion and relaxation in grossly inhomogeneous fields. J. Magn. 
Reson. 2002;157:31-42. doi: 10.1006/jmre.2002.2567.

67. Venkataramanan L, Song YQ, Hürlimann MD. Solving Fredholm integrals of the first kind with tensor product structure in 2 and 2.5 dimensions. IEEE Trans. Signal Process.

2002;50:1017-1026. doi: 10.1109/78.995059.

68. Galvosas P, Callaghan PT. Multi-dimensional inverse Laplace spectroscopy in the NMR of porous media. Comptes Rendus Phys. [Internet] 2010;11:172-180. doi:

10.1016/j.crhy.2010.06.014.

69. Ahola S, Zhivonitko V V., Mankinen O, Zhang G, Kantola AM, Chen HY, Hilty C, Koptyug I V., Telkki VV. Ultrafast multidimensional Laplace NMR for a rapid and sensitive chemical analysis. Nat. Commun. 2015;6. doi: 10.1038/ncomms9363.

70. Zhang Y, Blümich B. Spatially resolved D-T2 correlation NMR of porous media. J. Magn. Reson. [Internet] 2014;242:41-48. doi: 10.1016/j.jmr.2014.01.017.

71. Tax CM, Rudrapatna US, Witzel T, Jones DK. Disentangling in two dimensions in the living human brain: feasibility of relaxometry-diffusometry using ultra-strong gradients. In:

Proceedings of the 25th Annual Meeting of the International Society of Magnetic Resonance in Medicine (ISMRM). Honolulu, HI, USA; 2017. p. 838.

72. Kim D, Doyle EK, Wisnowski JL, Kim JH, Haldar JP. Diffusion-relaxation correlation spectroscopic imaging: A multidimensional approach for probing microstructure. Magn. Reson. Med. 2017;78:2236-2249. doi: 10.1002/mrm.26629.

73. Benjamini D, Basser PJ. Magnetic resonance microdynamic imaging reveals distinct tissue microenvironments. Neuroimage 2017;163:183-196. doi: 10.1016/j.neuroimage.2017.09.033.

74. Benjamini D, Basser PJ. Use of marginal distributions constrained optimization (MADCO) for accelerated 2D MRI relaxometry and diffusometry. J. Magn. Reson. 2016;271:40-45. doi: 10.1016/j.jmr.2016.08.004.

75. Le Bihan D, Breton E, Lallemand D, Aubin ML, Vignaud J, Laval-Jeantet M. Separation of diffusion and perfusion in intravoxel incoherent motion MR imaging. Radiology [Internet] 1988;168:497-505. doi: 10.1148/radiology.168.2.3393671.

76. Belliveau JW, Kennedy DN, McKinstry RC, Buchbinder BR, Weisskoff RM, Cohen MS, Vevea JM, Brady TJ, Rosen BR. Functional mapping of the human visual cortex by magnetic resonance imaging. Science (80-. ). [Internet] 1991;254:716-719. doi: 10.1126/science.1948051.

77. Kwong KK, Belliveau JW, Chesler DA, Goldberg IE, Weisskoff RM, Poncelet BP, Kennedy DN, Hoppel BE, Cohen MS, Turner R. Dynamic magnetic resonance imaging of human brain activity during primary sensory stimulation. Proc. Natl. Acad. Sci. U. S. A. [Internet] 1992;89:5675-5679. doi: $10.1073 /$ pnas.89.12.5675.

78. Ogawa S, Lee TM, Kay AR, Tank DW. Brain magnetic resonance imaging with contrast dependent on blood oxygenation. Proc. Natl. Acad. Sci. U. S. A. [Internet] 1990;87:9868-72. doi: 10.1073/pnas.87.24.9868. 
79. Zhong J, Kennan RP, Fulbright RK, Gore JC. Quantification of intravascular and extravascular contributions to BOLD effects induced by alteration in oxygenation or intravascular contrast agents. Magn. Reson. Med. 1998;40:526-536. doi: 10.1002/mrm.1910400405.

80. Darquié a, Poline JB, Poupon C, Saint-Jalmes H, Le Bihan D. Transient decrease in water diffusion observed in human occipital cortex during visual stimulation. Proc. Natl. Acad. Sci. U. S. A. [Internet] 2001;98:9391-5. doi: 10.1073/pnas.151125698.

81. Le Bihan D, Urayama S, Aso T, Hanakawa T, Fukuyama H. Direct and fast detection of neuronal activation in the human brain with diffusion MRI. Proc. Natl. Acad. Sci. U. S. A. [Internet] 2006;103:8263-8. doi: 10.1073/pnas.0600644103.

82. Tsurugizawa T, Ciobanu L, Le Bihan D. Water diffusion in brain cortex closely tracks underlying neuronal activity. Proc. Natl. Acad. Sci. U. S. A. [Internet] 2013;110:11636-11641. doi: 10.1073/pnas.1303178110.

83. Tsurugizawa T, Abe Y, Le Bihan D. Water Apparent Diffusion Coefficient Correlates With Gamma Oscillation of Local Field Potentials in the Rat Brain Nucleus Accumbens Following Alcohol Injection. J. Cereb. Blood Flow Metab. 2016;in press. doi: 10.1177/0271678X16685104.

84. Miller KL, Bulte DP, Devlin H, Robson MD, Wise RG, Woolrich MW, Jezzard P, Behrens TEJ. Evidence for a vascular contribution to diffusion FMRI at high b value. Proc. Natl. Acad. Sci. U. S. A. [Internet] 2007;104:20967-20972. doi: 10.1073/pnas.0707257105.

85. Bai R, Stewart C V., Plenz D, Basser PJ. Assessing the sensitivity of diffusion MRI to detect neuronal activity directly. Pnas [Internet] 2016:1519890113-. doi: 10.1073/pnas.1519890113.

86. Bai R, Klaus A, Bellay T, Stewart C, Pajevic S, Nevo U, Merkle H, Plenz D, Basser PJ. Simultaneous calcium fluorescence imaging and MR of ex vivo organotypic cortical cultures: $A$ new test bed for functional MRI. NMR Biomed. 2015;28:1726-1738. doi: 10.1002/nbm.3424.

87. Williams RJ, Reutens DC, Hocking J. Influence of BOLD contributions to diffusion fMRI activation of the visual cortex. Front. Neurosci. 2016;10. doi: 10.3389/fnins.2016.00279. 


\section{Figures}

Bloch's Equations with Diffusion Terms

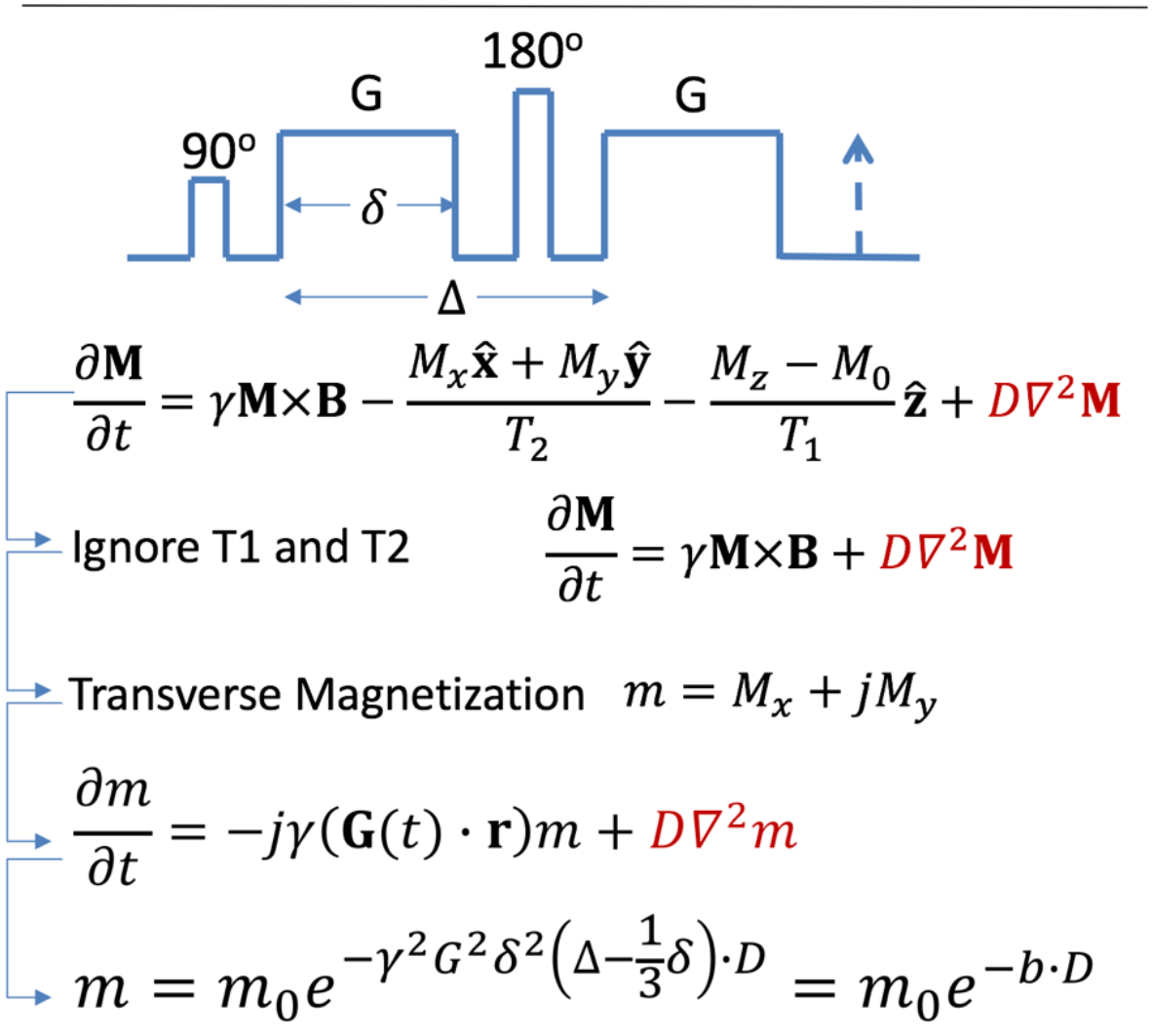

Figure 1. Overview of Bloch's equations with diffusion terms for a pulsed-gradient spin echo sequence. The diffusion terms are highlighted in red. 


\section{Ensemble Average of Spins with Random Phase}

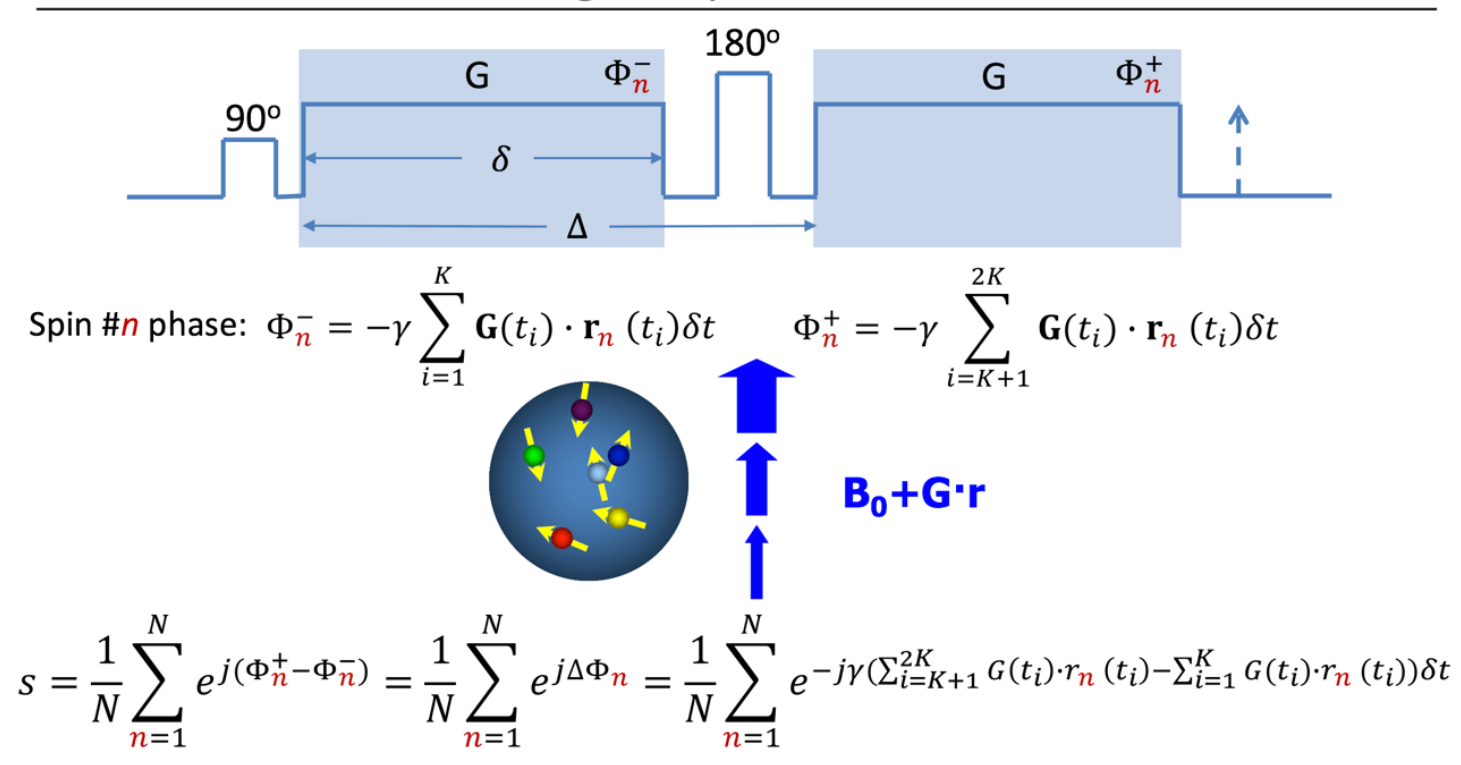

Figure 2. Overview of signal evaluation by ensemble average of spins with random phase accumulation due to diffusion in a gradient field. 


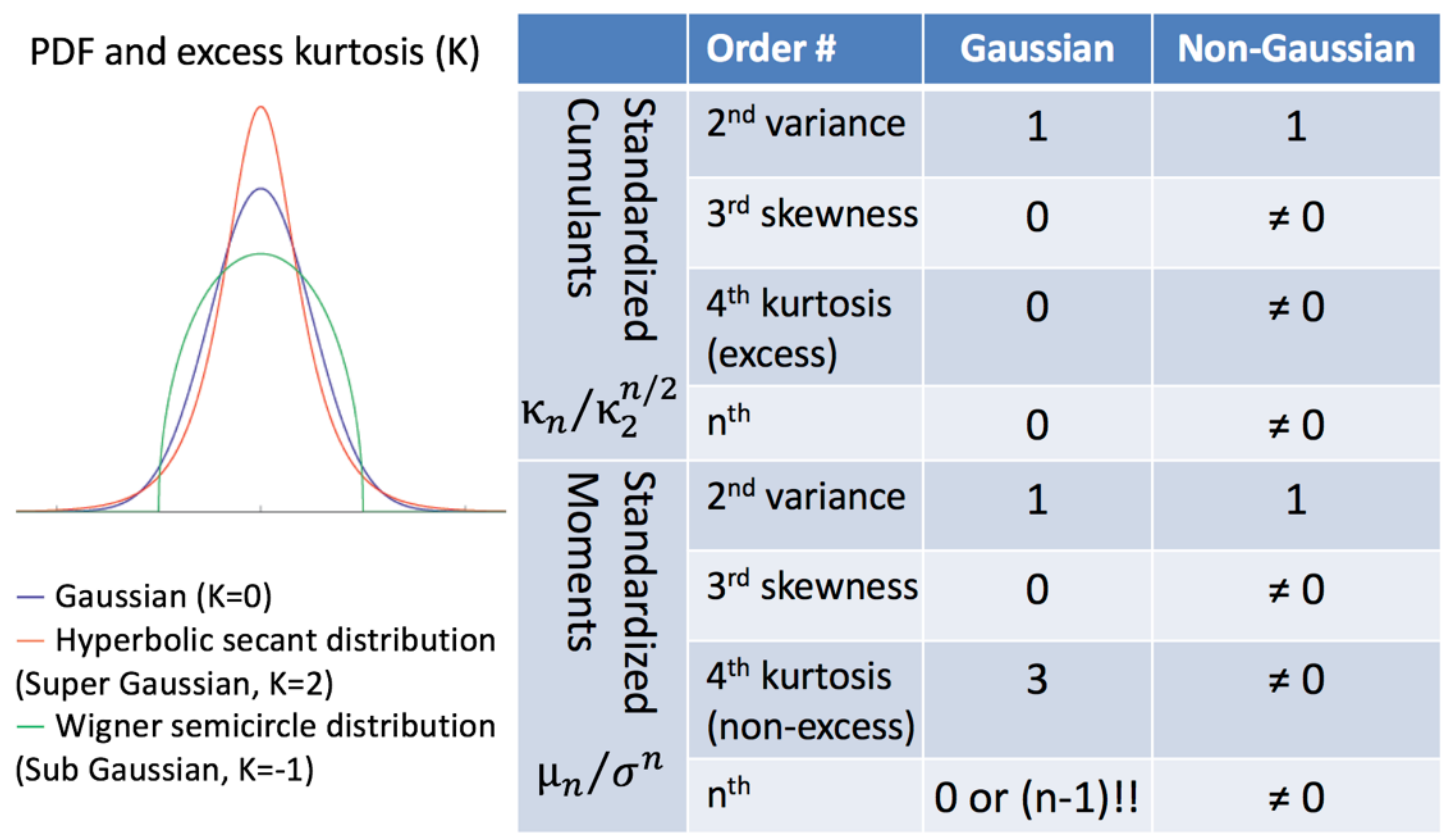

Figure 3. Comparison of higher order statistical properties of Gaussian vs. non-Gaussian diffusion processes. Left shows three examples of probability distribution function and corresponding excess kurtosis; right shows a table of standardized cumulants and moments of Gaussian and non-Gaussian distributions. Notice that the Gaussian distribution has non-zero higher-order (higher than 2) standardized moments but not cumulants. 


$$
\begin{array}{c|c}
\text { Generalized Bloch-Torrey } & \text { Statistical Approach } \\
\begin{array}{c|c}
\frac{\partial m}{\partial t}=-j \gamma(\mathbf{G} \cdot \mathbf{r}) m+D_{i_{1} i_{2}}^{(2)} \partial_{i_{1}} \partial_{i_{2}} m+D_{i_{1} i_{2} i_{3}}^{(3)} \partial_{i_{1}} \partial_{i_{2}} \partial_{i_{3}} m+\cdots & \langle s\rangle=\int e^{-i 2 \pi \mathbf{q} \cdot \mathbf{r}} p(\mathbf{r}) d \mathbf{r} \\
\frac{m}{m_{0}}=\exp \left(\sum_{n=2}^{\infty} j^{n} b_{i_{1} i_{2} \cdots i_{n}}^{(n)} D_{i_{1} i_{2} \cdots i_{n}}^{(n)}\right) & \langle s\rangle=\exp \left(\sum_{n=2}^{\infty}(-j)^{n} \frac{K_{i_{1} i_{2} \cdots i_{n}}^{(n)} q_{i_{1}} q_{i_{2}} \cdots q_{i_{n}}}{n !}\right) \\
D_{i_{1} i_{2} \cdots i_{n}}^{(n)}: n \text {-th order diffusion tensor } & K_{i_{1} i_{2} \cdots i_{n}}^{(n)}: n \text {-th order cumulant tensor of } \mathbf{r} \\
\hline & K_{i_{1} i_{2} \cdots i_{n}}^{(n)}=(-1)^{n} n ! D_{i_{1} i_{2} \cdots i_{n}}^{(n)}\left(\Delta-\frac{n-1}{n+1} \delta\right)
\end{array} \\
\text { Higher order diffusion tensors are measures of variance, skewness, kurtosis etc. }
\end{array}
$$

Figure 4. Diffusion signal equation derived from the Bloch-Torrey equation and statistical approaches. Comparing the two results, one may derive the relationship between cumulants and higher order diffusion tensors. 
$\langle s\rangle=\exp \left(\sum_{n=2}^{\infty}(-j)^{n} \frac{K_{i_{1} i_{2} \cdots i_{n}}^{(n)} q_{i_{1}} q_{i_{2}} \cdots q_{i_{n}}}{n !}\right) \Longrightarrow \begin{aligned} & \text { Asymmetrical structure requires odd-order } \\ & \text { cumulants ( } n \text { is odd), thus phase in DWI. }\end{aligned}$

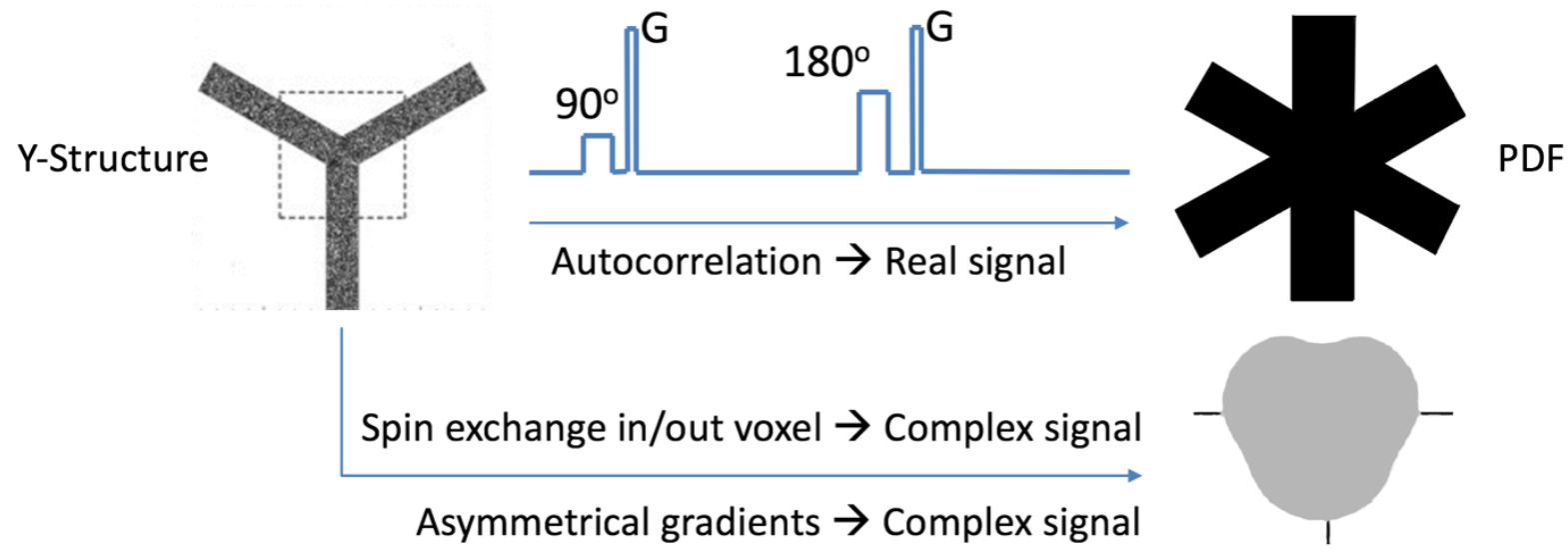

Figure 5. The need of phase information to reconstruct an asymmetrical structure. 


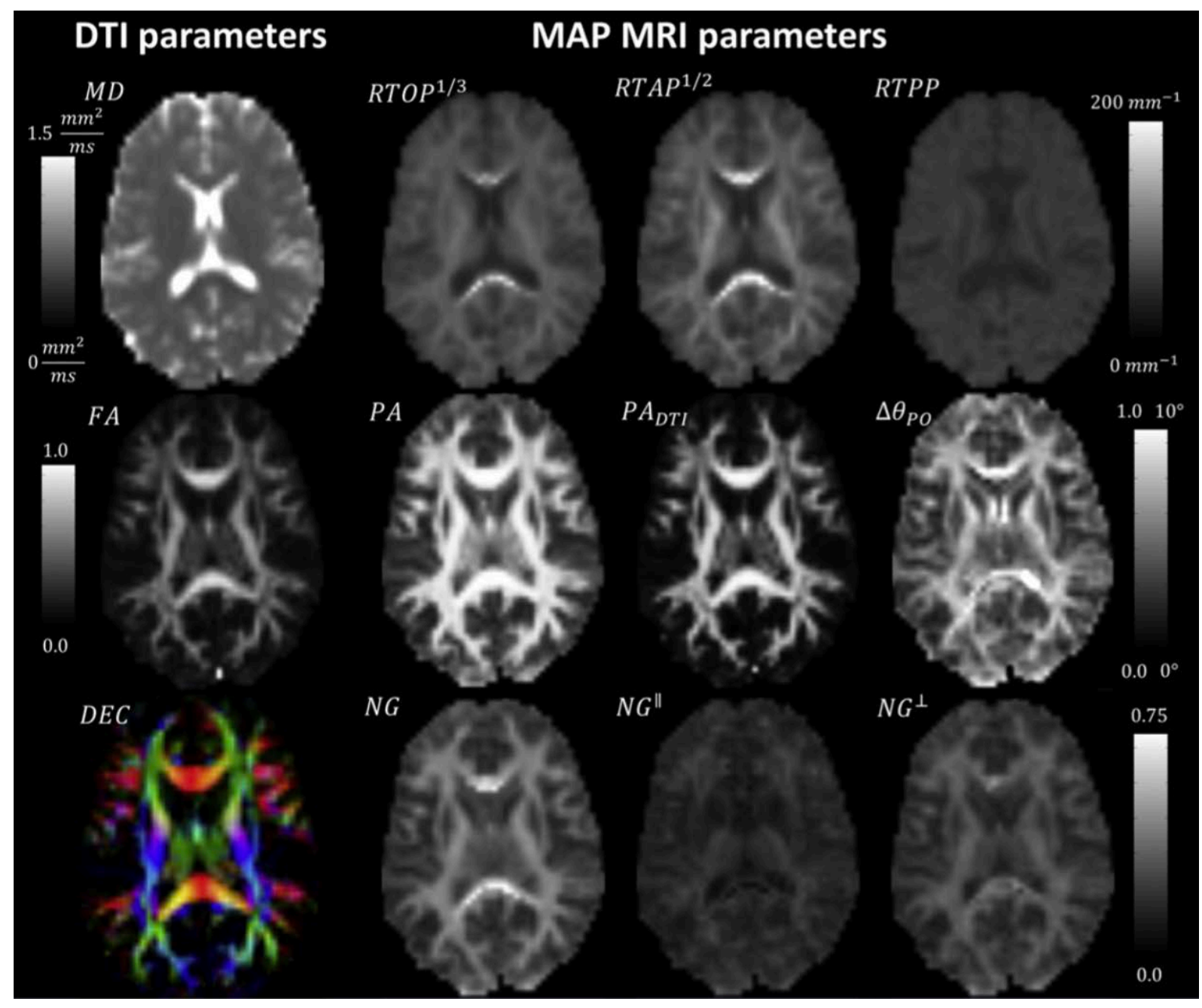

Figure 6. DTI-based parameters of mean diffusivity (MD), fractional anisotropy (FA), and direction-encoded color (DEC) maps are given on the left column. The remaining images are derived through the MAP-MRI framework. On the top row are the return-to-the-origin probability (RTOP), and its variants for diffusion parallel and perpendicular to the principal diffusion orientation - the return-to-the-plane probability (RTPP), and the return-to-the-axis probability (RTAP), respectively. The second row includes the propagator anisotropy (PA) measure followed by a comparable quantity for its Gaussian part. The enhancement of anisotropy due to non-Gaussian diffusion is illustrated via the rightmost map in the second row. Finally, on the last row are the non-Gaussianity (NG) index followed by its decomposition into directions parallel and perpendicular to the principal diffusion orientation. Adapted from Avram et al (24) with permission. 


\section{Restricted diffusion}
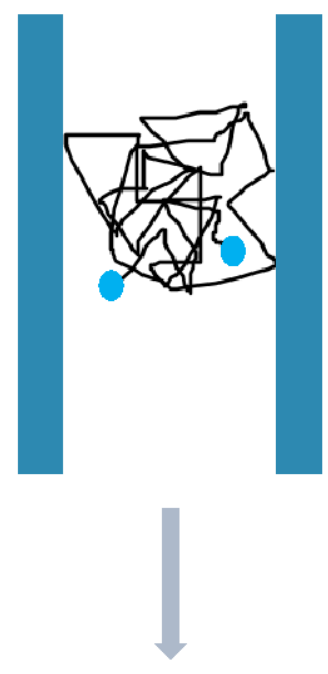

\section{Confinement model
(molecules subject to Hooke's force) \\ Confinement model
(molecules subject to Hooke's force)}

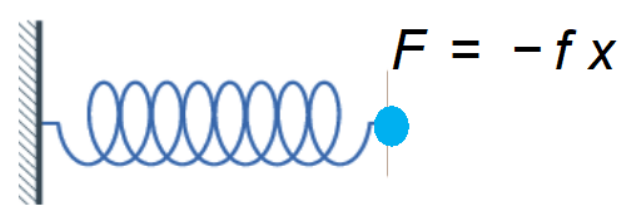

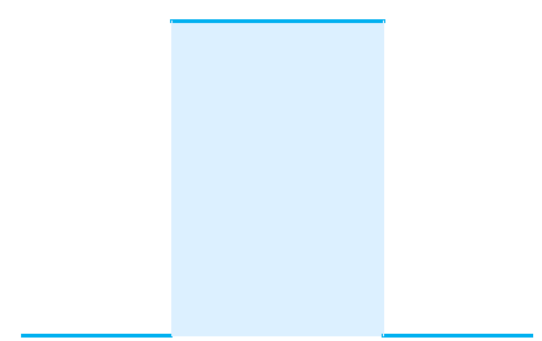

\section{Confinement: $C=f / k_{\mathrm{B}} T$}

\section{Distribution of particles at equilibrium}

Figure 7. The restricted diffusion process (top, left) can be restated as diffusion taking place within a potential energy profile, which takes a constant value within the pore space, and diverges at the walls. The equilibrium density is then constant within the pore, and vanishes at the walls (bottom, left). Many of the features of the restricted diffusion process are captured when one assumes a Gaussian equilibrium distribution (bottom, right). This distribution emerges for the problem involving particles subject to a Hookean restoring force (top, right). Here the potential is quadratic as a function of the distance $(x)$ from the attractive center of the pore. The confinement value is defined to be related to the force constant by a factor of $k_{B} T$, where $k_{B}$ is the Boltzmann constant and $T$ is the absolute temperature (25). Both models represent restricted diffusion in the sense that the mean squared displacements are bounded. Under certain experimental conditions, the Gaussianity inherent to the confinement model is approached by the restricted diffusion process (28). 


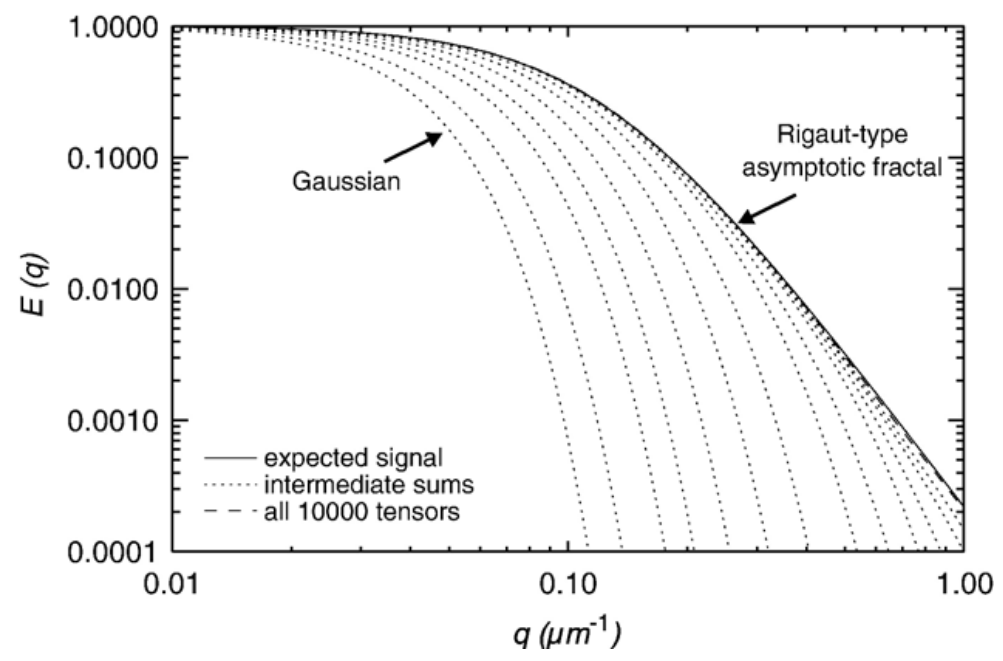

Figure 8. A simulation of the normalized signal intensity. The signal for only one compartment is a Gaussian. We gradually add the contributions from compartments with decreasing diffusivity. The power-law tail is obtained when all 10,000 tensors drawn from a Wishart distribution are included. Adapted from Jian et al. (29). 


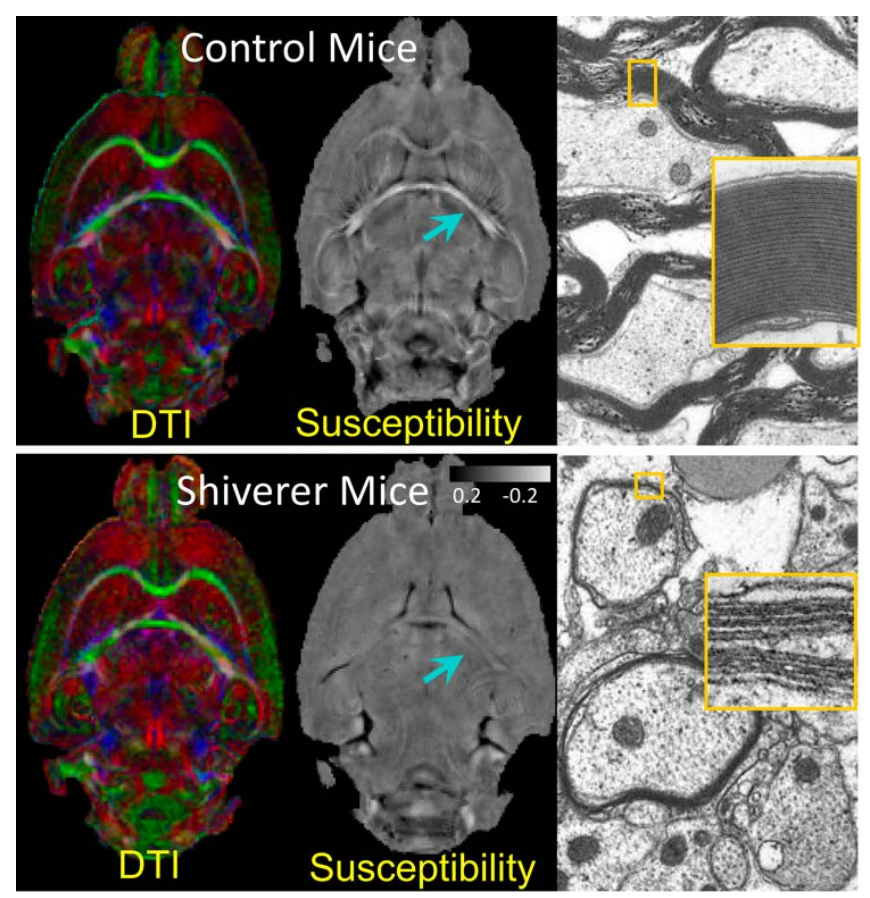

Figure 9. Dysmyelination resulting in total loss of susceptibility contrast between gray and white matter but a small loss of diffusion anisotropy. Adapted from Liu et al (47) with permission. 

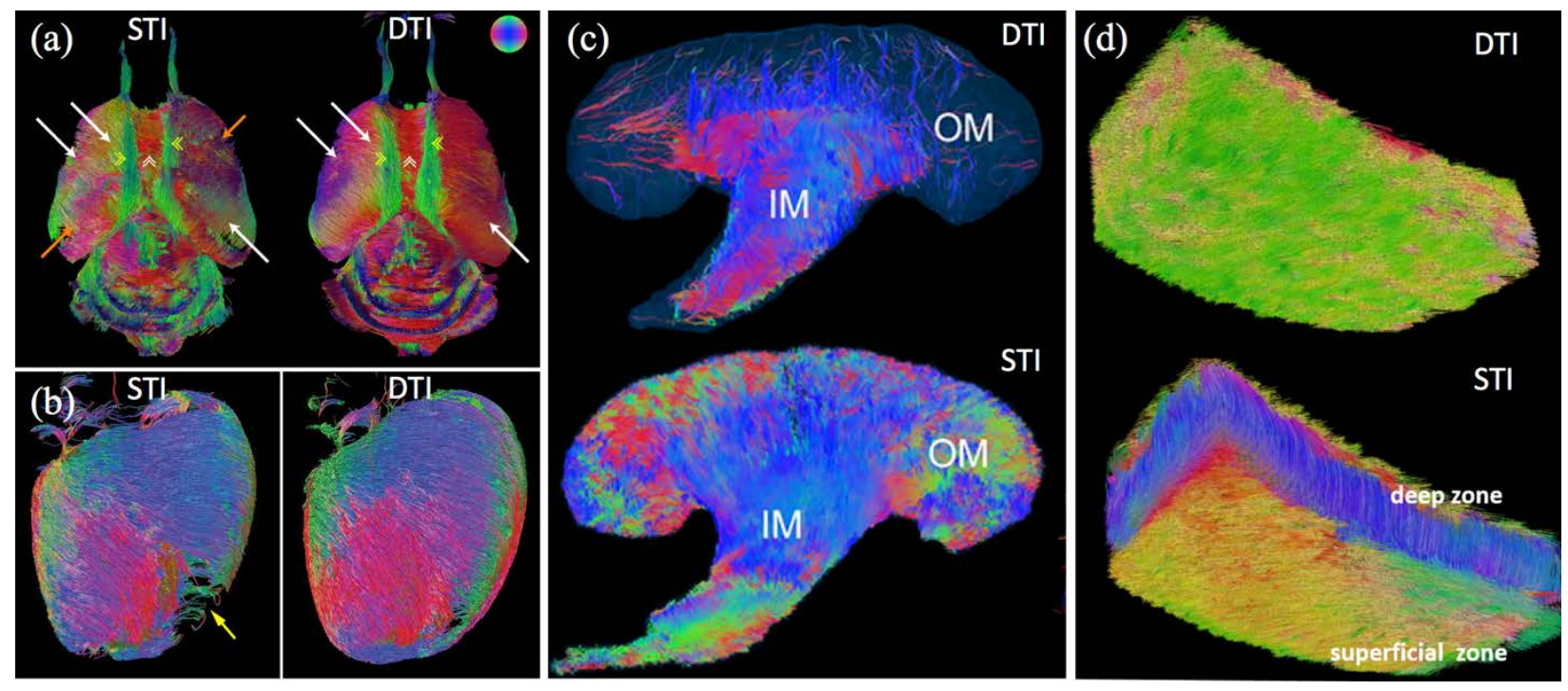

Figure 10. Comparisons of STI and DTI fiber tracking in (a) mouse brain, (b) mouse heart, (c) mouse kidney and (d) pig cartilage. 

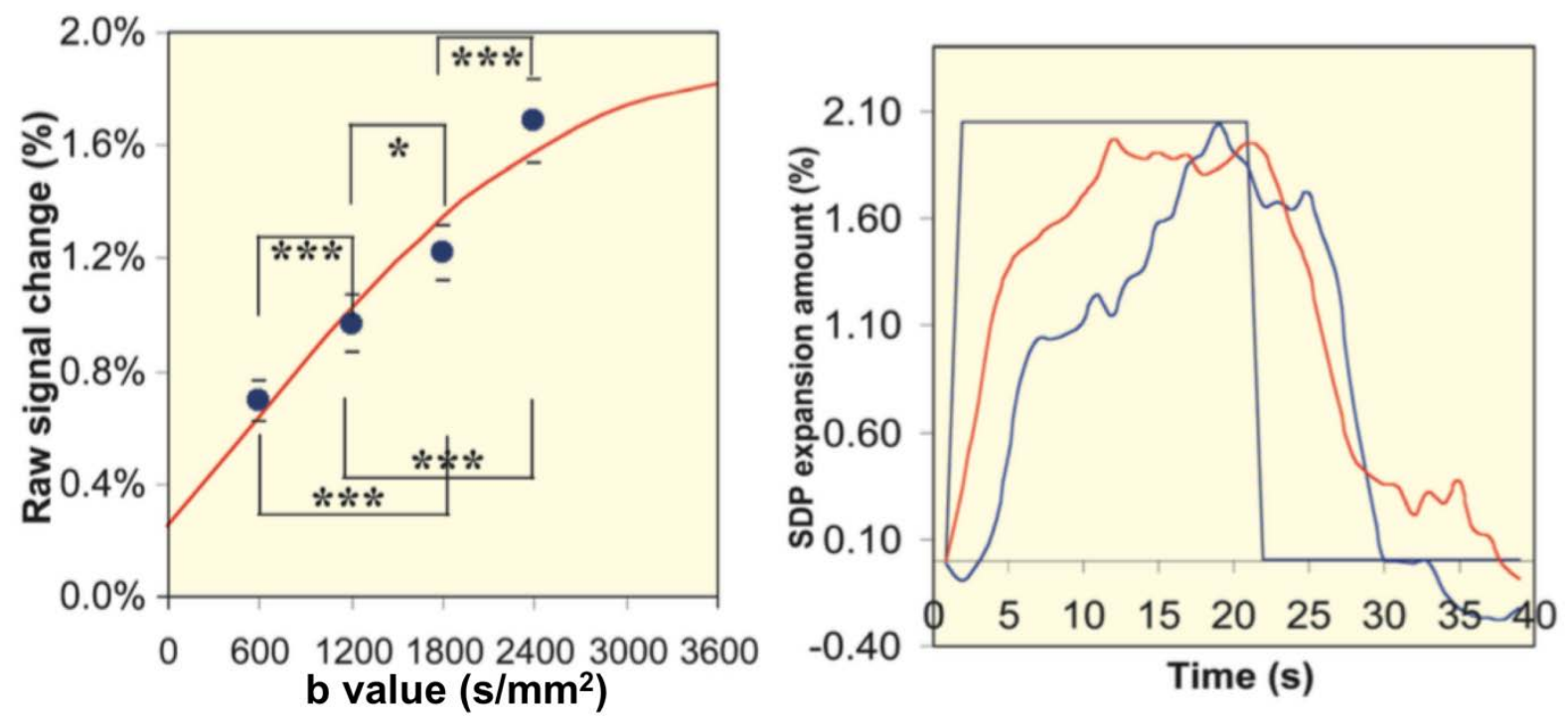

Figure 11. Left: The signal change associated with functional activation in diffusion-weighted acquisitions with varying diffusion sensitivity. The signal increase was assessed via statistical $t$ test by pairwise comparisons of the signal at different $b$ values and found to be significant. The marks *,**, and $* * *$ correspond to cases $P<0.05, P<0.01$, and $P<0.001$, respectively. Right: The black rectangular line indicates the time interval during which visual stimulus was induced by showing the patient the projection of a flickering dartboard. The red and blue curves depict the time courses of the slow diffusion phase (SDP) obtained from the diffusion signal values and the same for the BOLD fMRI signal, respectively. Figure adopted from Le Bihan et al. (81) with permission. 

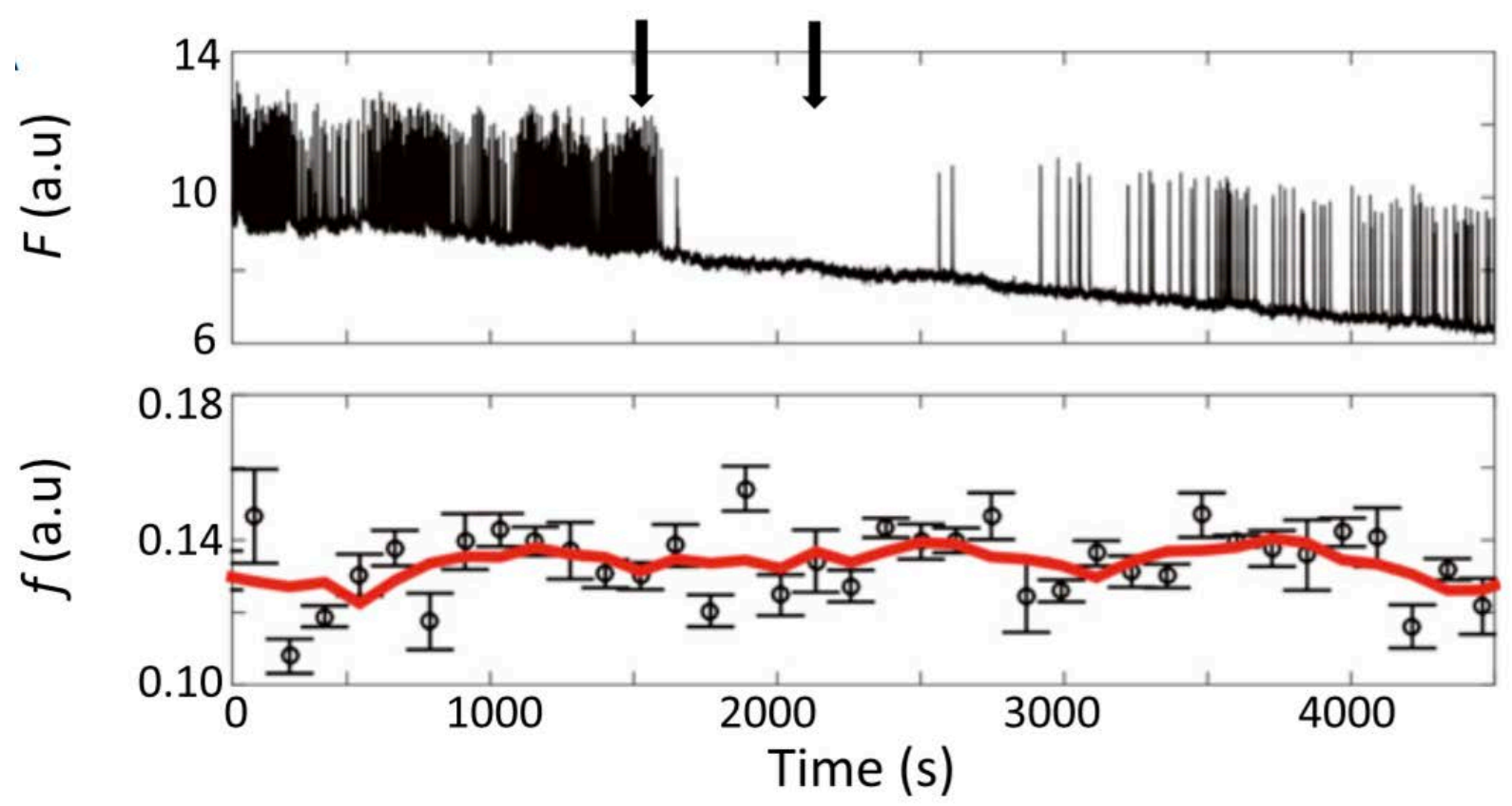

Figure 12. Neuronal activity is suppressed via TTX as shown by the calcium fluorescence signal on the top. The arrow on the left indicates the start of the TTX perfusion while the one on the right shows the washout with normal artificial cerebrospinal fluid (ACSF). The simultaneous measurement of the slow diffusion component of the signal is shown in the second row. The red curve is obtained by performing the average of six nearest data points. Figure adapted from Bai et al. (85) with permission. 